\title{
ASSESSMENT OF CHRONIC PANCREATITIS COURSE SEVERITY AND COMBINED MEDICAL TREATMENT USING PANCREATIN, PROTON PUMP INHIBITOR AND NON-STEROIDAL ANTI-INFLAMMATORY DRUG
}

Summary of the Doctoral Thesis to obtain doctoral degree of a Doctor of Medicine Speciality - Internal Medicine 
Larisa Umnova

\section{ASSESSMENT OF CHRONIC PANCREATITIS COURSE SEVERITY AND COMBINED MEDICAL TREATMENT USING PANCREATIN, PROTON PUMP INHIBITOR AND NON-STEROIDAL ANTI-INFLAMMATORY DRUG}

Summary of the Doctoral Thesis to obtain doctoral degree of a Doctor of Medicine Speciality - Internal Medicine 
Promotion work has been carried out at Rīga Stradiņš University (RSU)

Department of Internal Diseases

Scientific supervisor:

Dr. habil. med., Professor Grigorijs Orḷikovs,

RSU Department of Internal Diseases

Scientific adviser:

Dr. phys., Assistant Professor Renārs Erts,

RSU Department of Physics

Official reviewers:

Dr. med., Assistant Professor Aldis Pukīitis,

University of Latvia, The Faculty of Medicine,

Department of Internal Medicine

Dr. med., Professor Jāzeps Keišs, Latvian Infectology Centre

Dr. habil. med., Professsor Arunas Savickas,

Lithuanian University of Health Sciences, Department of Drugs

technology and social pharmacy, Kaunas, Lithuania

The Doctoral Thesis will be defended on the $3^{\text {rd }}$ of November, 2014 at 15.00 at an open meeting of Promotion Council of Medicine of Rīga Stradinš University at the Hippocrates Lecture Theatre, in 16 Dzirciema Street, Riga.

The Doctoral Thesis is available at the library Rīga Stradiņš University and homepage: www.rsu.lv

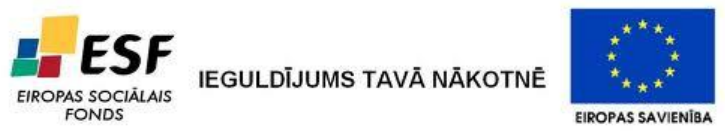

The Doctoral Thesis was supported by the ESF co-financed project

"Support to doctoral students for obtaining the study program and acquiring the scientific degree at Rīga Stradinnš University", agreement No. 2009/0147/1DP/1.1.2.1.2/09/IPIA/VIAA/009.

Secretary of the promotion council:

Dr. habil. med., Professor Maija Eglīte 


\section{CONTENTS}

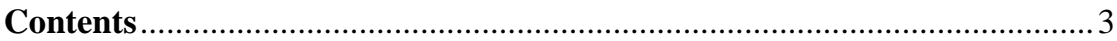

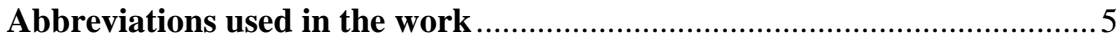

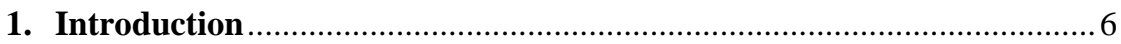

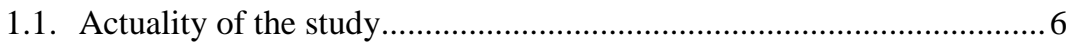

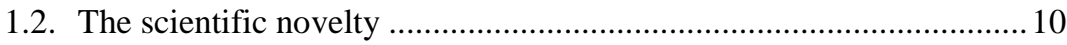

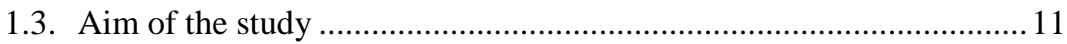

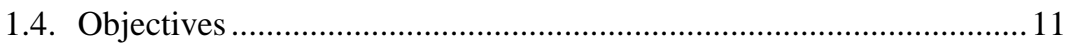

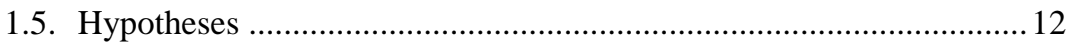

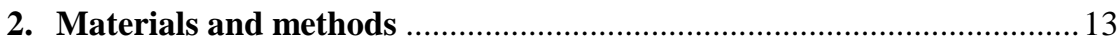

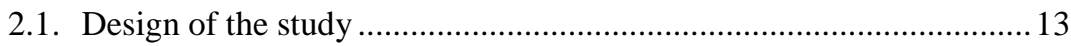

2.2. Investigations before and after medical treatment course ................... 15

2.3. Abdominal pain, bowel movements and appetite loss assessment ..... 16

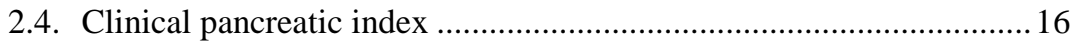

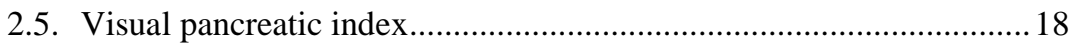

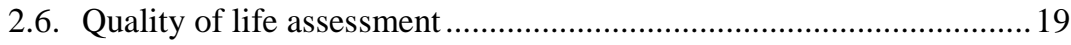

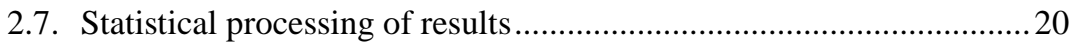

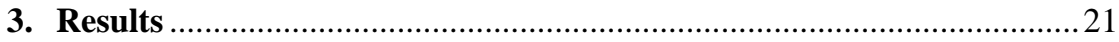

3.1. Data of clinical examination, laboratory and radiologic

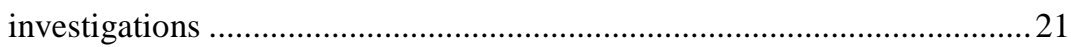

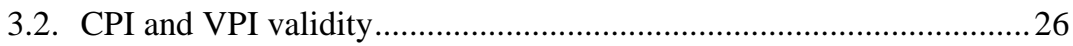

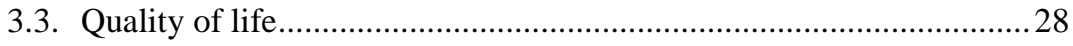

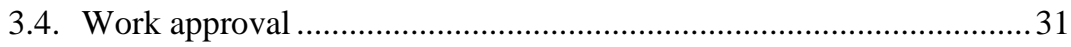




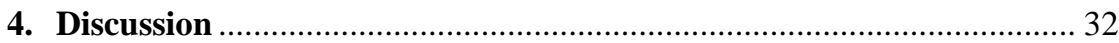

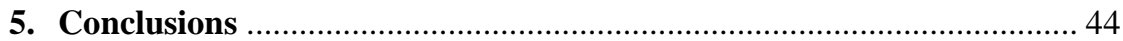

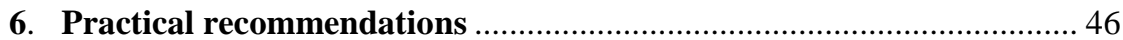

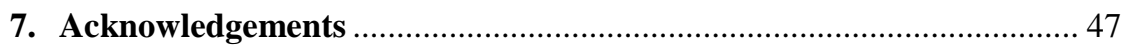

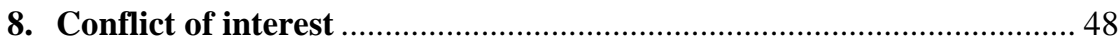

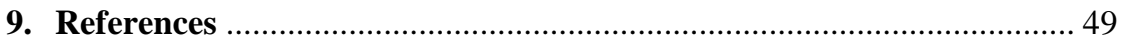

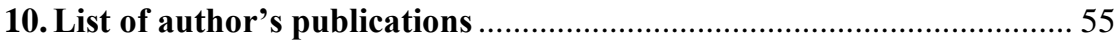




\section{ABBREVIATIONS USED IN THE WORK}
ALAT - alanine aminotransferase
ASAT - aspartate aminotransferase
BMI - body mass index
CCK - cholecystokinin
COX - cyclooxygenase
CPI - Clinical pancreatic index
CT - computed tomography

EORTC QLQ-C30 - European Organization for Research and Treatment of Cancer Quality of Life Questionnaire - C30

ESR - erythrocyte sedimentation rate

EUS - endoscopic ultrasonography

FE-1 - faecal elastase 1

FU - EORTC QLQ-C30 functional scales sum of points

GH - EORTC QLQ-C30 global health scale

$\mathrm{CP}$ - chronic pancreatitis

NSAID - non-steroidal anti-inflammatory drug

$\mathrm{P}$ - pancreatin

PNP - pancreatin, a proton pump inhibitor and a non-steroidal antiinflammatory drug combination

PPI - proton pump inhibitor

QLQ-PAN26 - European Organization for Research and Treatment of Cancer Quality of Life Questionnaire Pancreatic Cancer Module

QoL - quality of life

SY - EORTC QLQ-C30 symptom scales sum of points

USG - ultrasonography

VPI - Visual pancreatic index 


\section{INTRODUCTION}

Chronic pancreatitis (CP) is a chronic pancreas exocrine part inflammation which is accompanied by organ parenchyma destruction and fibrosis [1]. Alcohol consumption and smoking are the most common CP risk factors. The illness is ever progressing while often causing severe complications [2]. The given disease is likely to have combinations such as pancreas cancer and high morbidity level [3]. CP notably reduces patient working capacity [4], social activity, quality of life (QoL) and lifetime duration. $\mathrm{CP}$ remains latent for a long time. Clinical symptoms and results of laboratory investigation are convincing only during disease exacerbation or in case of a long term illness. Timely diagnosis of CP is quite complicated, but very urgent in terms of stopping the progression of the disease.

\subsection{Actuality of the study}

There are no certain data about CP prevalence in Latvia. The cases of acute and chronic pancreatitis are considered together [5, 6]. According to the data of World Health Organization, alcohol consumption in Latvia corresponds to the average European levels, which are considered to be the two times higher than in the world - 10.4-14.5 litres of pure alcohol consumed per person aged 15 years or older [7]. Considering that $\mathrm{CP}$ etiological variations are not common in Latvia, it is supposed that alcohol caused $\mathrm{CP}$ is the most widespread etiological variation. Smoking is a co-factor in CP etiology, and, according to WHO, Latvia has high rates of smoking [8]. Taking into account these indices, it can be assumed that CP prevalence and incidence are identical to the data of European countries - 26-27 cases out of 100000 inhabitants.

One of current problems in $\mathrm{CP}$ treatment is such that there is a lack of a precise and trustworthy disease severity and therapy effectiveness assessment 
method $[9,10]$. Many currently known CP classifications reflect stages of the disease [11], but do not show CP clinical course severity, even though it is exactly the CP clinical symptom and pancreas structural change severity that defines the choice of therapy method. In turn, CP clinical classifications are not easy to use and are rarely applied in $\mathrm{CP}$ clinical studies and in practice. Nowadays a further work on disease clinical classification is of priority. CP classification as a tool for $\mathrm{CP}$ clinical severity assessment must include criteria for a quantitative and qualitative assessment of all important disease clinical symptoms. CP classification must be easy to use. It must be able to reflect both $\mathrm{CP}$ activity in every period of time and the effectiveness of the treatment without bias. At the moment such CP classification is not available yet.

$\mathrm{CP}$ was considered a surgical pathology for a long time. Even though $\mathrm{CP}$ patients receive an effective surgical [12] and endoscopic [13] treatment, those methods are not always necessary and a medical treatment remains the main CP curing way. Usually the goal of CP medical therapy is the reduction of disease clinical symptoms, pancreas exocrine and endocrine function substitution. The main CP clinical symptoms are abdominal pain and malabsorbtion. At the moment widely accepted medical therapy tactic is not elaborated. Standards of disease treatment suggest a rather symptomatic CP therapy with a choice of medications step by step depending on the effect reached $[14,15,16]$. Pancreatins (animal pancreas extracts), analgetics, somatostatin analogues, antidepressants, antioxidants and other medications are suggested to cure $\mathrm{CP}$ with, but their role in $\mathrm{CP}$ treatment is not yet fully clear. Many researches were made in order to figure out the effectiveness of those preparations, but the results of studies and their conclusions are contradictory [17].

At the moment the one of the most popular $\mathrm{CP}$ treatment methods is the use of pancreatins. Pancreatin, while acting on CCK, reduces pancreas activity, provides analgesic effect $[18,19]$ and provides the substitution of 
exocrine function [20]. The analgesic effect of pancreatin for CP patients is not approved in all researches [21, 22, 23, 24], what can be explained by different research design, heterogeneity of patient population in studies, different pancreatins use and other factors. The current task is to ascertain the type of $\mathrm{CP}$ patient groups that could have a convincing analgesic effect. For example, for patients with small duct $\mathrm{CP}[25]$ and preserved pancreas exocrine secretion a pancreatin can have a high analgesic effect, because in this case pancreatin can reach inhibitive effect on pancreas exocrine secretion basing on feedback principle [26]. Owing to that, there are sound opportunities of applying pancreatins for $\mathrm{CP}$ abdominal pain reduction.

Pancreatins do not reduce secretin effect - the production of pancreas bicarbonates and water. This effect can theoretically be reached through inhibition of of proton pumps $\left(\mathrm{H}^{+} / \mathrm{K}^{+}\right.$-ATPase), using proton pump inhibitor preparations. In a recent study [27] about rat pancreas ducts an $86 \%$ reduction of secretin stimulated bicarbonate and water production due to omeprazol was demonstrated. This discovery is relevant to a case of human $\mathrm{CP}$ treatment. This means that PPI could have a bigger role in $\mathrm{CP}$ medical treatment.

One of the main CP pathophysiological mechanisms is pancreas inflammation $[28,29]$, but there is a small amount of studies about NSAID role in CP therapy [30]. It is now important to continue the researches related to NSAID capabilities not only in the area of abdominal pain, but pancreas inflammation and fibrosis reduction. It is now unclear, if NSAID application is safe for CP patients.

When curing $\mathrm{CP}$, it is now essential to figure out if, while simultaneously influencing three basic pathophysiological mechanisms of $\mathrm{CP}$ pancreas protheolytic activity increase [31], inflammation and exocrine insufficiency - it is possible to reach a better effect on CP clinical course. In given study a PNP therapy that considers pancreatin, PPI and NSAID combination (Fig. 1.1.) was elaborated. 


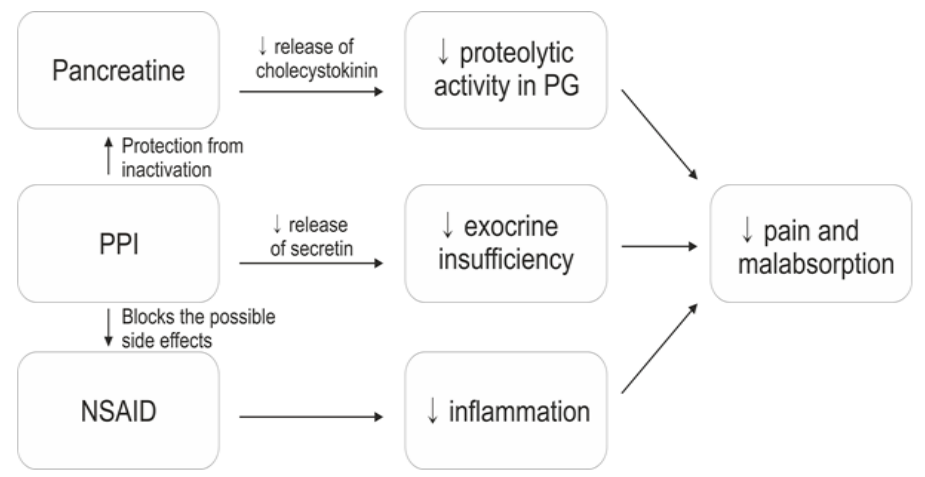

Figure 1.1. Pattern of PNP therapy of chronic pancreatitis $(\downarrow-$ decrease)

PNP therapy is supported by three principles. First one - every PNP combination component affects pain mechanisms independently. Second - if two or three preparations are combined, PNP components fight pathophysiological CP mechanisms in synergy. Third - PPI blocks possible side effects of NSAID [32, 33, 34].

The use of PNP therapy provides a reasonable hope for an effective reduction of main $\mathrm{CP}$ pathogenic symptom - abdominal pain, pancreas inflammation sign reduction and exocrine insufficiency correction. Pancreatin, PPI and NSAID simultaneous combination in curing CP is pathophysiologically reasoned and can be more effective compared to pancreatin monotherapy and pancreatin and PPI combination. Considering that pancreatin, PPI and NSAID combined therapy usefulness and safety for CP patients has never been studied before, it is a timely research.

Assessment of quality of life (QoL), while reflecting the CP patient's well-being fairly, can be a useful additional therapy effectiveness assessment method [35]. QoL assessment for CP patients was successfully applied, evaluating the effectiveness of antioxidant therapy and surgical treatment [12, 36, 37]. In CP studies QoL is a timely method that helps in figuring out the effectiveness of treatment and gives an opportunity to compare the results of 
the studies. Only a small amount of CP studies that used QoL assessment were done in Latvia previously.

\subsection{The scientific novelty}

For the first time specially elaborated quantitative methods - Clinical pancreatic index (CPI) and Visual pancreatic index (VPI) - were used to assess $\mathrm{CP}$ pancreas structural change severity and the effectiveness of medical treatment. On 22.02.2012 VPI was registered as an invention in Patent office of the Republic of Latvia (patent Nr. LV 14515 B) named „Method of defining indications to surgical interference in case of chronic pancreatitis" [38].

For the first time in Latvia and the world a research to assess medical therapy effectiveness involving pancreatin, PPI and NSAID combination for $\mathrm{CP}$ patients was completed. For the first time a combined medical therapy of pancreatin, PPI and NSAID was justified and applied for CP patients who did not have indications for a surgical or endoscopic treatment.

For the first time in Latvia $\mathrm{CP}$ patients before and after medical treatment course had their quality of life assessed with EORTC QLQ-C30 and QLQ-PAN26.

The scientific novelty of the study demonstrated in fifteen scientific articles and one invention registered in Patent office of the Republic of Latvia. The study results demonstrated in four international congress and conferences as well as in four Latvian conferences. 


\subsection{Aim of the study}

To develop new assessment methods of chronic pancreatitis (CP) clinical course severity and pancreas structural change severity, and using pancreatin as monotherapy and as combination with proton pump inhibitor (PPI), as well as a combination of pancreatin, PPI and NSAID in patients with $\mathrm{CP}$, to define the most effective medication treatment way among the ones applied.

\subsection{Objectives}

1. To elaborate a Clinical pancreatic index (CPI) for an integral quantitative assessment of CP clinical course severity and study CPI capabilities for assessment of $\mathrm{CP}$ clinical course severity in patients who receive pancreatin monotherapy ( $\mathrm{P}$ therapy), pancreatin and proton pump inhibitor combination (PP therapy) and pancreatin, proton pump inhibitor and nonsteroidal anti-inflammation drug combination (PNP therapy).

2. To elaborate a Visual pancreatic index (VPI) for an integral quantitative assessment of pancreas structural changes and study the VPI utility in CP patients who receive $\mathrm{P}, \mathrm{PP}$ and PNP therapy.

3. To study pancreatin P, PP and PNP therapy impact on CP patient's complaints, laboratory and radiological investigations results.

4. To study P, PP and PNP therapy impact on CP patient's quality of life.

5. To compare P, PP and PNP therapy impact on CP patient's complaints, results of laboratory and radiology research, disease clinical course severity and quality of life and determine the most effective therapy method. 


\subsection{Hypotheses}

1. Integral quantitative assessment of body mass loss, abdominal pain, steatorrhea, daily bowel movements, other dyspeptic complaints, glucose tolerance and some patient's anamnesis data objectively represents $\mathrm{CP}$ clinical course and medical treatment efficiency.

2. Integral quantitative assessment of pancreas head size, echotexture, calcinates, diameter of ductus pancreaticus, pseudocysts, pancreas contours, peripancreatic fibrosis objectively represents pancreas changes severity in case of CP.

3. For patients with CP pancreatin, proton pump inhibitor and non-steroidal anti-inflammation drug combination is more effective compared to pancreatin monotherapy and pancreatin combination with proton pump inhibitor. 


\section{MATERIALS AND METHODS}

\subsection{Design of the study}

The given work is elaborated in Centre of Gastroenterology of Paul Stradins university hospital between 2007 and 2013. The study is completed basing on permission of Ethics committee of Rīga Pauls Stradiňš University and corresponds to European directive "Good clinical practice" and the „Declaration of Helsinki”. Pangrol, Omeprazol and Airtal medications from BERLIN-CHEMIE, OlainFarm un Gedeon Richter companies were received in the form of humanitarian aid. One hundred and eighteen patients with $\mathrm{CP}$ were investigated in prospective controlled randomized study. A reexamination had one hundred and six patients $(89.8 \%)$. A duration of a treatment was one month.

Patient inclusion criteria:

- patients with confirmed diagnosis of $\mathrm{CP}$, who did not require surgical or endoscopic treatment;

- patients of both sexes, the age of patients was between 20 and 76 years;

- patients participation agreement.

Patient exclusion criteria:

- patients with malignant diseases;

- pacients with gastric or duodenum ulcer;

- patients with gastrointestinal bleeding in anamnesis;

- patients with infectious diseases, pacients with severe liver and kidney diseases;

- patients with acute cerebral or myocardial infarction, patients with significant heart failure; 
- patients with mental diseases;

- patients who disagree with alcohol abstinence during the study.

Before a treatment indications for surgery such as bile duct obstruction, duodenal stenosis, left-sided portal hypertension, big pancreatic pseudocysts, ascites, hydrothorax, and pain resistant to medication were ruled out. Patient division between research groups see in Tab. 2.1.

Table 2.1.

Division of patients and types of therapy

\begin{tabular}{|l|c|c|}
\hline \multicolumn{1}{|c|}{ Group } & Type of medical treatment & $\begin{array}{c}\text { Number of } \\
\text { patients }\end{array}$ \\
\hline P & Pangrol $25000-1$ capsule t.i.d. & 20 \\
\hline PP & $\begin{array}{r}\text { Pangrol } 25000-1 \text { capsule t.i.d. } \\
\text { Omeprazols } 20 \mathrm{mg} \text { pa } 1 \text { capsule b.i.d. }\end{array}$ & 48 \\
\hline PNP & $\begin{array}{r}\text { Pangrol } 25000-1 \text { capsule t.i.d. } \\
\text { Omeprazols } 20 \mathrm{mg}-1 \text { capsule b.i.d. }\end{array}$ & 38 \\
\hline Total & Airtal (aceclofenac) $100 \mathrm{mg}-1$ tab. b.i.d. & 106 \\
\hline
\end{tabular}

Pangrol 25000 is enteric coated microspheres of pancreatin. Every Pangrol 25000 capsule lipase activity corresponds to 25000 EPU (European Pharmacopoeia units), amylase activity not less than 22500 EPU and protease activity not less than 1250 EPU. Pangrol 25000 manufacturer - BERLINCHEMIE AG (MENARINI GROUP). Every Omeprazols capsule contains 20 $\mathrm{mg}$ of omeprazole in hard gelatine capsule. „Olainfarm” JSC produced omeprazol was used for research. Airtal is a coated pill that contains $100 \mathrm{mg}$ of aceclofenac, manufacturer - Gedeon Richter.

The final analysis included 68 males and 38 females:

- sex-ratio between males and females was 2:1;

- average age of patients (SD) was 53.33 (12.42) years;

- average disease duration: median $=36$; IQR $=78$ month; 
- before the study 63 patients consumed alcohol regularly (59.4\%) - 48 males (70.6\% of males) and 15 females (39.5\% of males);

- 54 patients were smokers $(50.9 \%)$ - 46 males (67.6\% of males) and 7 females (18.4\% of females);

- in 2 patients (1.89\%) the cause of CP was hypertriglyceridemia;

- 103 pacients (97.2\%) had abdominal pain;

- small duct CP stated for $84,5 \%$ research patients, these patients had ductus pancreaticus diameter of $3.5 \mathrm{~mm}$ and less.

\subsection{Investigations before and after medical treatment course:}

Before and after one month long therapy the following tests were conducted to patient:

- assessment of patient's complaints including abdominal pain, bowel movements and appetite, history taking, physical examination, BMI $(\mathrm{kg} / \mathrm{m} 2)$;

- blood analysis: blood Hb, RBC and WBC count, ESR (standard methods);

- blood biochemical tests - glucose, calcium, ASAT, ALAT, $\alpha$-amylase, urea (standard methods);

- fecal microscopy - steatorrhea (fecal microscopy), benzidine test;

- fecal elastase-1 (FE-1) (ELISA polyclonal antibody method);

- abdominal ultrasonoscopy or computed tomography;

- assessment of quality of life according to EORTC QLQ-C30 and QLQPAN26;

- before treatment all patients had fibrogastroscopy.

Research was entirely completed in Pauls Stradins Clinical University Hospital Center of Gastroenterology, excluding FE-1 definition. FE-1 was defined in Clinical immunology centre Immunology laboratory of Pauls Stradins Clinical University Hospital and in Immunology laboratory BIOCON. 


\subsection{Abdominal pain, bowel movements and appetite loss assessment}

During treatment, patients made daily notes of abdominal pain, bowel movements and appetite loss, basing on specially designed criteria (see Tab. 2.2.).

Table 2.2.

Criteria for abdominal pain, bowel movements and appetite loss assessment

\begin{tabular}{|l|c|c|}
\hline \multicolumn{1}{|c|}{ Symptom } & Degree of the symptom & Points \\
\hline \multirow{3}{*}{$\begin{array}{l}\text { Abdominal pain (modified } \\
\text { visual analogue scale) }\end{array}$} & severe pain $(8-10$ points by VAS $)$ & 3 \\
\cline { 2 - 3 } & moderate pain $(4-7$ points by VAS $)$ & 2 \\
\cline { 2 - 3 } & mild $(1-3$ points by VAS $)$ & 1 \\
\cline { 2 - 3 } & no pain & 0 \\
\hline \multirow{3}{*}{ Bowel movements } & diarrhea & 2 \\
\cline { 2 - 3 } & constipation & 1 \\
\hline \multirow{2}{*}{ Loss of appetite } & normal & 0 \\
\cline { 2 - 3 } & yes & 0 \\
\hline
\end{tabular}

Analysis included the sums of the first and the last five days' points of abovementioned symptoms. In order to assess abdominal pain, a standard 10 cm visual analogue scale (VAS) was used. Modified results were used in analysis, which were classified as mild, moderate and severe pain.

\subsection{Clinical pancreatic index}

The clinical course of CP was assessed using a quantitative indicant Clinical pancreatic index (CPI) (see Tab. 2.3.). CPI is based on CP severity criteria. CPI implies an assessment and summarization of such CP severity indicators as number of surgical procedures due to $\mathrm{CP}$ complications in 
anamnesis, number of hospital courses for CP, weight loss, abdominal pain severity, steatorrhea severity, daily bowel movements, other dyspeptic complaints, and glucose intolerance.

Table 2.3.

\section{CP clinical course severity assessment criteria (CPI)}

\begin{tabular}{|c|c|c|}
\hline Criterion & Degree/number & Points \\
\hline \multirow{4}{*}{$\begin{array}{l}\text { Number of surgical procedures due to } \mathrm{CP} \\
\text { complications in anamnesis }\end{array}$} & no & 0 \\
\hline & one & 1 \\
\hline & two & 2 \\
\hline & three and more & 3 \\
\hline \multirow{2}{*}{ Efficiency of outpatient treatment } & effective & 0 \\
\hline & ineffective & 2 \\
\hline \multirow{4}{*}{$\begin{array}{l}\text { Number of hospitalizations due to CP in } \\
\text { anamnesis }\end{array}$} & no & 0 \\
\hline & one & 1 \\
\hline & two & 2 \\
\hline & three and more & 3 \\
\hline \multirow{4}{*}{ Loss of weight (kg) } & $0 \mathrm{~kg}$ & 0 \\
\hline & less than $3 \mathrm{~kg}$ & 1 \\
\hline & $3-5 \mathrm{~kg}$ & 2 \\
\hline & $>5 \mathrm{~kg}$ & 3 \\
\hline \multirow{4}{*}{$\begin{array}{l}\text { Severity of pain VAS (Visual Analogue } \\
\text { Scale) }\end{array}$} & no pain & 0 \\
\hline & mild & 1 \\
\hline & moderate & 2 \\
\hline & severe & 3 \\
\hline \multirow{4}{*}{ Degree of steatorrhea } & 0 or + & 0 \\
\hline & ++ & 1 \\
\hline & +++ & 2 \\
\hline & ++++ and more & 3 \\
\hline \multirow{4}{*}{ Number of daily defecations } & no & 0 \\
\hline & one & 1 \\
\hline & two & 2 \\
\hline & three and more & 3 \\
\hline \multirow{4}{*}{$\begin{array}{l}\text { Other dyspeptic complaints (abdominal } \\
\text { bloating, borborygmus, nausea, } \\
\text { postprandial abdominal discomfort) }\end{array}$} & no & 0 \\
\hline & one & 1 \\
\hline & two & 2 \\
\hline & three and more & 3 \\
\hline \multirow{3}{*}{ Glucose tolerance } & normal & 0 \\
\hline & impaired & 1 \\
\hline & diabetes mellitus & 2 \\
\hline
\end{tabular}


The higher CPI, the more severe is the clinical course of CP. Maximum CPI score is 25 (see Tab. 2.3). Depending on the score, severity of $\mathrm{CP}$ clinical course is assessed as follows: mild $-6-8$ points, moderate $-9-15$, severe $-16-25$ points. CPI reflects doctor's opinion regarding a severity of CP clinical course.

\subsection{Visual pancreatic index}

For quantitative pancreas structural change assessment a Visual pancreatic index (VPI) was developed (see Tab. 2.4.).

Table 2.4.

\section{Criteria of pancreas parenchyma structure changes in case of CP (VPI)}

\begin{tabular}{|c|c|c|}
\hline Criterion & Degree of expressiveness & Points \\
\hline \multirow{4}{*}{ The size of caput pancreatic } & $3.0 \mathrm{~cm}$ & 0 \\
\hline & $>3.0 \mathrm{~cm}$ & 1 \\
\hline & $2.5-2.9 \mathrm{~cm}$ & 2 \\
\hline & $<2.5 \mathrm{~cm}$ & 3 \\
\hline \multirow{4}{*}{ The echotexture of pancreas } & homogenic & 0 \\
\hline & medium granular & 1 \\
\hline & local granular & 2 \\
\hline & diffuse granular & 3 \\
\hline \multirow{4}{*}{ Calcinates } & no calcinates & 0 \\
\hline & local small & 1 \\
\hline & multiple 3-4 $\mathrm{mm} \varnothing$ & 2 \\
\hline & diffuse & 3 \\
\hline \multirow{4}{*}{$\begin{array}{l}\text { Maximal diameter of } \\
\text { ductus pancreaticus }\end{array}$} & $<3 \mathrm{~mm}$ & 0 \\
\hline & $3-4 \mathrm{~mm}$ & 1 \\
\hline & $4.1-5.0 \mathrm{~cm}$ & 2 \\
\hline & $5 \mathrm{~mm}$ & 3 \\
\hline \multirow{4}{*}{ Pseudocysts } & no pseudocysts & 0 \\
\hline & $<3 \mathrm{~cm} \varnothing$ & 1 \\
\hline & $3.1-5 \mathrm{~cm} \mathrm{Ø}$ & 2 \\
\hline & $>5 \mathrm{~cm} \varnothing$ & 3 \\
\hline \multirow{4}{*}{ Other changes } & no changes & 0 \\
\hline & irregular contours of pancreas & 1 \\
\hline & peripancreatic fibrosis & 2 \\
\hline & concrements in ductus pancreaticus & 3 \\
\hline
\end{tabular}


VPI is based on 6 pancreas visual parameter groups: pancreas head size, pancreas echostructure, calcinates, ductus pancreaticus diameter, pseudocysts and other. CP pancreas changes were classified depending on severity - total score: $0-3$ points - CP diagnosis is doubtful; $4-8$ points mild changes; $9-14$ points - moderate changes; $15-21$ - severe changes. Abdominal ultrasonography data was used for calculation of VPI; however for VPI calculation other sources can be used, such as endoscopic ultrasonography, abdominal CT, endoscopic retrograde cholangeopancreatography.

$\mathrm{CPI}$ and VPI reflect the severity of $\mathrm{CP}$, both indexes are quantitative, easy to use and, what is more, invasive and expensive researches are not required to calculate them.

\subsection{Quality of life assessment}

In order to evaluate the quality of life, EORTC-QLQ-C30 and QLQPAN26 were used. With the help of EORTC-QLQ-C30 the patient's physical (PF), emotional (EF), cognitive (CF), social (SF), and role functioning (RF), general health and quality of life were assessed, as well as such CP symptoms as fatigue (FA), nausea/vomiting (NV), pain (PA), dyspnoea (DY), sleep disturbance (SL), appetite loss (AP), constipation (CO), diarrhea (DI) and financial difficulties (FI). In total there were 30 simple questions in the EORTC QLQ-30 questionnaire. The worse the patients rated their functional status and QoL, the lower was the rate. The stronger the $\mathrm{CP}$ symptoms, the higher were the corresponding index. In order to assess the general functioning of the patient, the EORTC QLQ-C30 functioning points were summed up (FU), and in order to assess the severity of the patient's CP symptoms, all EORTC QLQC30 symptom points were summed up (SY). The QLQ-PAN26 questionnaire was used to assess the additional symptoms of CP and the QoL (abdominal pain, digestive problems, jaundice, bowel movements disorder, flatulence, 
muscle weakness, dry mouth, treatment side effects, weight loss, anxiety about the future health, opportunity to plan life and others). The EORTC QLQ-C30 and QLQ-PAN26 are used in combination as a system, in order to achieve better results in evaluating the $\mathrm{QoL}$ of the $\mathrm{CP}$ patient. In total there are 56 questions in this questionnaire system.

\subsection{Statistical processing of results}

The statistical processing of research results was done using IBM SPSS 20.0 version. The analysis involved Wilcoxon Signed Ranks Test, MannWhitney Test. Considering multi-factor analysis with additional filtering parameters, more than 2 group analysis is done applying ANCOVA (Analysis of Covariance), because initial patient parameters are taken into consideration additionally; in order to define the difference of statistically valid groups Posthoc Sidac analysis was used. Statistical significance level $\mathrm{p}<0.05$ was considered in research. Still, statistical significance level $\mathrm{p}<0.1$ highlights a tendency that a statistically valid difference exists. In order to define the validity of CPI and VPI Kronbach alpha coefficient was calculated. Alpha values of 0.7 and higher are considered as acceptable. In order to figure out the most effective treatment, the following criteria were taken into account: statistically valid changes of every indicant after treatment $(\mathrm{p}<0.05)$; every indicant difference between groups after treatment (using ANCOVA analysis); visual presentation of parameters under research. 


\section{RESULTS}

\subsection{Data of clinical examination, laboratory and radiologic investigations}

Patients in group $\mathrm{P}$ experienced statistically proved improvements in issues of abdominal pain, appetite loss, WBC count, blood $\alpha$-amylase, ASAT, ALAT, and blood urea $(\mathrm{p}<0.05)$ (see Tab. 3.1.).

Table 3.1.

\section{Indicant changes in group $P$}

\begin{tabular}{|c|c|c|c|}
\hline Indicant & $\begin{array}{c}\text { Mean value before } \\
\text { treatment } \pm \\
\text { standart error }\end{array}$ & $\begin{array}{c}\text { Mean value after } \\
\text { treatment } \pm \\
\text { standart error }\end{array}$ & $\begin{array}{c}\mathrm{p} \\
\text { value* }\end{array}$ \\
\hline BMI $\left(\mathrm{kg} / \mathrm{m}^{2}\right)$ & $25.24 \pm 0.99$ & $26.43 \pm 1.13$ & 1.000 \\
\hline Abdominal pain \# & $9.21 \pm 0.96$ & $6.11 \pm 0.84$ & 0.008 \\
\hline CPI VAS $^{\nabla}$ & $2.33 \pm 0.29$ & $1.33 \pm 0.29$ & 0.047 \\
\hline Abnormal bowel movements ${ }^{\#}$ & $3.35 \pm 0.84$ & $2.19 \pm 0.67$ & 0.137 \\
\hline Loss of appetite $^{\#}$ & $2.33 \pm 0.52$ & $0.93 \pm 0.41$ & 0.035 \\
\hline Steatorrhea $^{\nabla}$ & $1.21 \pm 0.33$ & $1.00 \pm 0.21$ & 0.206 \\
\hline FE-1 $(\mu \mathrm{g} / \mathrm{g})$ & $179.20 \pm 42.60$ & $219.92 \pm 44.70$ & 0.327 \\
\hline WBC count $\left(10^{9} / \mathrm{L}\right)$ & $9588.00 \pm 642.00$ & $7475.00 \pm 304.00$ & 0.003 \\
\hline RBC count $\left(10^{12} / \mathrm{L}\right)$ & $4.44 \pm 0.18$ & $4.44 \pm 0.12$ & 0.625 \\
\hline $\mathrm{Hb}(\mathrm{g} / \mathrm{L})$ & $138.53 \pm 5.40$ & $135.23 \pm 4.18$ & 0.861 \\
\hline $\mathrm{ESR}(\mathrm{mm} / \mathrm{h})$ & $16.00 \pm 3.23$ & $15.50 \pm 3.09$ & 0.310 \\
\hline Blood $\alpha$-amylase (U/L) & $329.78 \pm 52.00$ & $135.92 \pm 26.00$ & 0.003 \\
\hline ALAT(U/L) & $70.00 \pm 12.20$ & $42.64 \pm 5.64$ & 0.005 \\
\hline $\operatorname{ASAT}(\mathrm{U} / \mathrm{L})$ & $87.25 \pm 21.30$ & $46.64 \pm 7.14$ & 0.004 \\
\hline Urea $(\mathrm{moll} / \mathrm{l})$ & $6.70 \pm 0.51$ & $5.50 \pm 0.39$ & 0.041 \\
\hline Glucose tolerance $^{\nabla}$ & $0.24 \pm 0.11$ & $0.07 \pm 0.07$ & 0.180 \\
\hline Total CPI $^{\nabla}$ & $10.05 \pm 1.02$ & $7.88 \pm 0.81$ & 0.068 \\
\hline Total $\mathrm{VPI}^{\Omega}$ & $5.60 \pm 1.19$ & $4.67 \pm 1.07$ & 0.581 \\
\hline
\end{tabular}

\#- sum of points according patient's diary; $\nabla$ - sum of points according CPI; ${ }^{\Omega}-$ sum of points according VPI; BMI - body mass index; VAS - visual analogue scale; ESR - erythrocyte sedimentation rate; ALAT - alanine aminotransferase; ASAT - aspartate aminotransferase; CPI clinical pancreatic index; VPI - visual pancreatic index; * - Wilcoxon test

Patients in group PP had statistically proved improvements in issues of abdominal pain, bowel movement disturbance, steatorrhea, blood $\alpha$-amylase, 
glucose intolerance, WBC count, ESR, ALAT, ASAT, CPI ( $\mathrm{p}<0.05)$ (see Tab. 3.2.).

\section{Indicant changes in group PP}

Table 3.2.

\begin{tabular}{|c|c|c|c|}
\hline Indicant & $\begin{array}{c}\text { Mean value } \\
\text { before treatment } \pm \\
\text { standard error }\end{array}$ & $\begin{array}{l}\text { Mean value after } \\
\text { treatment } \pm \\
\text { standard error }\end{array}$ & $\mathrm{p}$ value* \\
\hline BMI $\left(\mathrm{kg} / \mathrm{m}^{2}\right)$ & $24.99 \pm 0.91$ & $25.00 \pm 0.89$ & 0.671 \\
\hline Abdominal pain $^{\#}$ & $8.62 \pm 0.83$ & $5.62 \pm 0.84$ & 0.006 \\
\hline CPI VAS $^{\nabla}$ & $2.18 \pm 0.18$ & $1.18 \pm 0.18$ & 0.009 \\
\hline Abnormal bowel movements ${ }^{\#}$ & $3.10 \pm 0.82$ & $0.95 \pm 0.36$ & 0.016 \\
\hline Loss of appetite $^{\#}$ & $0.95 \pm 0.36$ & $0.57 \pm 0.33$ & 0.270 \\
\hline Steatorrhea ${ }^{\nabla}$ & $1.85 \pm 0.21$ & $0.40 \pm 0.13$ & $<0.001$ \\
\hline FE-1 $(\mu \mathrm{g} / \mathrm{g})$ & $240.93 \pm 54.40$ & $236.93 \pm 44.70$ & 0.286 \\
\hline WBC count $\left(10^{9} / \mathrm{L}\right)$ & $8779.00 \pm 590.00$ & $7435.00 \pm 494.00$ & 0.009 \\
\hline RBC count $\left(10^{12} / \mathrm{L}\right)$ & $4.66 \pm 0.10$ & $4.64 \pm 0.09$ & 0.324 \\
\hline $\mathrm{Hb}(\mathrm{g} / \mathrm{L})$ & $138.65 \pm 3.00$ & $137.10 \pm 2.00$ & 0.837 \\
\hline $\operatorname{ESR}(\mathrm{mm} / \mathrm{h})$ & $17.85 \pm 2.56$ & $13.65 \pm 1.87$ & 0.021 \\
\hline Blood $\alpha$-amylase (U/L) & $127.60 \pm 17.30$ & $83.25 \pm 9.44$ & 0.001 \\
\hline ALAT (U/L) & $30.45 \pm 4.75$ & $23.47 \pm 2.31$ & 0.025 \\
\hline ASAT (U/L) & $29.85 \pm 2.93$ & $26.37 \pm 2.39$ & 0.025 \\
\hline Urea (moll//l) & $6.82 \pm 0.61$ & $6.45 \pm 0.62$ & 0.295 \\
\hline Glucose tolerance $^{\nabla}$ & $0.65 \pm 0.18$ & $0.40 \pm 0.17$ & 0.025 \\
\hline 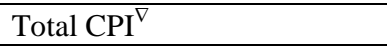 & $12.26 \pm 0.62$ & $5.33 \pm 0.52$ & $<0.001$ \\
\hline Total VPI ${ }^{\Omega}$ & $2.47 \pm 0.23$ & $2.11 \pm 0.25$ & 0.176 \\
\hline
\end{tabular}

For explanations see Tab. 3.1.

Patients in group PNP after treatment experienced statistically proved improvements in BMI, abdominal pain, bowel movements, appetite, steatorrhea, blood $\alpha$-amylase, WBC count, ESR, VPI, CPI $(\mathrm{p}<0.05)$ (see Tab. 3.3.). Indicant change significance level comparison between the study groups see tab. 3.4 . 
Table 3.3.

\section{Indicant changes in group PNP}

\begin{tabular}{|l|c|c|c|}
\hline \multicolumn{1}{|c|}{ Indicant } & $\begin{array}{c}\text { Mean value before } \\
\text { treatment } \pm \text { standard } \\
\text { error }\end{array}$ & $\begin{array}{c}\text { Mean value after } \\
\text { treatment } \pm \\
\text { standard error }\end{array}$ & p value* \\
\hline BMI $\left(\mathrm{kg} / \mathrm{m}^{2}\right)$ & $24.77 \pm 1.03$ & $25.33 \pm 1.05$ & $<0.001$ \\
\hline Abdominal pain $^{\#}$ & $9.78 \pm 0.61$ & $2.19 \pm 0.62$ & $<0.001$ \\
\hline CPI VAS $^{\nabla}$ & $2.42 \pm 0.13$ & $0.42 \pm 0.10$ & $<0.001$ \\
\hline $\begin{array}{l}\text { Abnormal bowel } \\
\text { movement }^{\#}\end{array}$ & $3.87 \pm 0.63$ & $0.53 \pm 0.27$ & $<0.001$ \\
\hline Loss of appetite $^{\#}$ & $2.33 \pm 0.41$ & $0.17 \pm 0.10$ & $<0.001$ \\
\hline Steatorrhea & $1.73 \pm 0.18$ & $0.31 \pm 0.09$ & $<0.001$ \\
\hline FE-1 $(\mu \mathrm{g} / \mathrm{g})$ & $206.43 \pm 36.00$ & $283.95 \pm 41.30$ & 0.070 \\
\hline WBC count $\left(10^{9} / \mathrm{L}\right)$ & $10325.00 \pm 776.00$ & $7448.00 \pm 324.00$ & 0.001 \\
\hline RBC count $\left(10^{12} / \mathrm{L}\right)$ & $4.87 \pm 0.10$ & $4.69 \pm 0.10$ & 0.215 \\
\hline Hb $(\mathrm{g} / \mathrm{L})$ & $146.43 \pm 3.70$ & $141.57 \pm 2.69$ & 0.100 \\
\hline ESR $(\mathrm{mm} / \mathrm{h})$ & $21.45 \pm 3.65$ & $11.43 \pm 1.88$ & 0.018 \\
\hline Blood $\alpha$-amylase $(\mathrm{U} / \mathrm{L})$ & $343.46 \pm 75.90$ & $99.97 \pm 17.60$ & $<0.001$ \\
\hline ALAT $(\mathrm{U} / \mathrm{L})$ & $53.50 \pm 13.60$ & $37.83 \pm 6.50$ & 0.721 \\
\hline ASAT $(\mathrm{U} / \mathrm{L})$ & $54.58 \pm 12.00$ & $31.00 \pm 5.00$ & 0.600 \\
\hline Urea $(\mathrm{mmol} / \mathrm{l})^{\text {Glucose tolerance }}{ }^{\nabla}$ & $4.91 \pm 0.57$ & $5.45 \pm 0.54$ & 0.500 \\
\hline Total CPI & $0.48 \pm 0.13$ & $0.39 \pm 0.13$ & 0.317 \\
\hline Total VPI ${ }^{\Omega}$ & $12.00 \pm 0.66$ & $2.87 \pm 0.37$ & $<0.001$ \\
\hline & $5.28 \pm 0.59$ & $3.70 \pm 0.54$ & $<0.001$ \\
\hline
\end{tabular}

For explanations see Tab. 3.1. 
Table 3.4.

Indicant change significance level comparison between study groups

\begin{tabular}{|c|c|c|c|}
\hline \multirow[t]{2}{*}{ Indicant } & \multicolumn{3}{|c|}{$\begin{array}{c}\text { Difference between indicant average before and } \\
\text { after treatment }\end{array}$} \\
\hline & group $\mathrm{P}$ & group PP & group PNP \\
\hline $\mathrm{BMI}\left(\mathrm{kg} / \mathrm{m}^{2}\right)$ & -1.19 & -0.01 & $-0.56 * *$ \\
\hline Abdominal pain ${ }^{\#}$ & $3.10 *$ & $3.00 *$ & $7.59 * *$ \\
\hline CPI VAS $^{\nabla}$ & $1.00 *$ & $1.00 *$ & $2.00 * *$ \\
\hline Abnormal bowel movement ${ }^{\#}$ & 1.16 & $2.15^{*}$ & $3.34 * *$ \\
\hline Loss of appetite $^{\#}$ & $1.40 *$ & 0.38 & $2.16^{* *}$ \\
\hline Steatorrhea $^{\nabla}$ & 0.21 & $1.45 * *$ & $1.42 * *$ \\
\hline Fecal elastase $1(\mu \mathrm{g} / \mathrm{g})$ & -40.72 & 4.00 & -77.52 \\
\hline WBC count $(109 / \mathrm{L})$ & $2113.00 *$ & $1344.00 *$ & $2877 *$ \\
\hline RBC count $\left(10^{12} / \mathrm{L}\right)$ & 0.00 & 0.02 & 0.18 \\
\hline $\mathrm{Hb}(\mathrm{g} / \mathrm{L})$ & 3.30 & 1.55 & 4.86 \\
\hline $\operatorname{ESR}(\mathrm{mm} / \mathrm{h})$ & 0.50 & $4.20 *$ & $10.02 *$ \\
\hline Blood $\alpha$-amylase (U/L) & $193.86^{*}$ & $44.35 *$ & $243.49 * *$ \\
\hline ALAT (U/L) & $27.36^{*}$ & $6.98 *$ & 15.67 \\
\hline ASAT (U/L) & $40.64 *$ & $3.48 *$ & 23.58 \\
\hline Urea $(\mathrm{mmol} / \mathrm{l})$ & $1.20 *$ & $0.37 *$ & -0.54 \\
\hline Glucose tolerance $^{\nabla}$ & 0.17 & $0.25 *$ & 0,09 \\
\hline Total $\mathrm{CPI}^{\nabla}$ & 2.17 & $6.93 * *$ & $9.13 * *$ \\
\hline Total $\mathrm{VPI}^{\Omega}$ & 0.93 & 0.36 & $1.58 * *$ \\
\hline
\end{tabular}

${ }^{\#}$ - sum of points according patient's diary; ${ }^{\nabla}$ - sum of points according CPI; ${ }^{\Omega}$ - sum of points according VPI; $*-\mathrm{p}<0.05 ; * *-\mathrm{p}<0.001$

Such CP therapy safety indicants as RBC count and $\mathrm{Hb}$ remained unchanged after treatment in all groups of patients; moreover, they were similar after treatment in all groups. In P and PP groups ASAT and ALAT level decreased significantly $(\mathrm{p}<0.05)$ after treatment, while in PNP group they remained unchanged. Blood urea showed a drop only in group $\mathrm{P}(\mathrm{p}=0.041)$; however in PP and PNP groups no such changes were noticed.

While analysing CPI and VPI changes after treatment, a notable CPI reduction is noticed in PP and PNP groups ( $p<0.001$ ), in turn, VPI statistically notably changed only for PNP group patients $(\mathrm{p}<0.001)$. 
Table 3.5.

\section{Clinical and additional investigation indicant comparison between study groups}

\begin{tabular}{|c|c|c|c|c|}
\hline \multirow[t]{2}{*}{ Indicant } & \multirow{2}{*}{$\begin{array}{l}\text { Three groups } \\
\text { comparison } \\
\text { (ANCOVA } \\
\text { test, } \mathrm{p} \text { value) }\end{array}$} & \multicolumn{3}{|c|}{$\begin{array}{c}\text { Indicant after treatment } \\
\text { comparison (ANCOVA Post-hoc } \\
\text { Sidac test, } \mathrm{p} \text { value) }\end{array}$} \\
\hline & & $\begin{array}{c}\mathrm{P} \text { and } \\
\text { PP }\end{array}$ & $\begin{array}{l}\mathrm{P} \text { and } \\
\text { PNP }\end{array}$ & $\begin{array}{l}\text { PP and } \\
\text { PNP }\end{array}$ \\
\hline BMI $\left(\mathrm{kg} / \mathrm{m}^{2}\right)$ & 0.019 & 0.234 & 0.070 & 0.068 \\
\hline Abdominal pain ${ }^{\#}$ & $<0.001$ & 0.389 & $<0.001$ & $<0.001$ \\
\hline CPI VAS $^{\nabla}$ & 0.001 & 0.540 & 0.001 & 0.004 \\
\hline Abnormal bowel movement ${ }^{\#}$ & 0.020 & 0.033 & 0.006 & 0.628 \\
\hline Loss of appetite ${ }^{\#}$ & 0.056 & 0.337 & 0.022 & 0.161 \\
\hline Steatorrhea $^{\nabla}$ & 0.001 & 0.003 & $<0.001$ & 0.526 \\
\hline Fecal elastase $1(\mu \mathrm{g} / \mathrm{g})$ & 0.366 & 0.336 & 0.872 & 0.177 \\
\hline WBC count $(109 / \mathrm{L})$ & 0,669 & 0.940 & 0.521 & 0.410 \\
\hline RBC count $\left(10^{12} / \mathrm{L}\right)$ & 0.770 & 0.512 & 0.510 & 0.988 \\
\hline $\mathrm{Hb}(\mathrm{g} / \mathrm{L})$ & 0.629 & 0.575 & 0.339 & 0.648 \\
\hline $\operatorname{ESR}(\mathrm{mm} / \mathrm{h})$ & 0,170 & 0.137 & 0.610 & 0.565 \\
\hline Blood $\alpha$-amylase (U/L) & 0.086 & 0.197 & 0.029 & 0.328 \\
\hline ALAT (U/L) & 0.438 & 0.032 & 0.642 & 0.213 \\
\hline $\operatorname{ASAT}(\mathrm{U} / \mathrm{L})$ & 0.084 & 0.031 & 0.137 & 0.582 \\
\hline Urea $(\mathrm{mmol} / \mathrm{l})$ & 0.241 & 0.094 & 0.411 & 0.518 \\
\hline Glucose tolerance $^{\nabla}$ & 0.130 & 0.418 & 0.054 & 0.183 \\
\hline Total $\mathrm{CPI}^{\nabla}$ & $<0.001$ & 0.019 & $<0.001$ & 0.001 \\
\hline Total VPI $^{\Omega}$ & 0.271 & 0.344 & 0.113 & 0.480 \\
\hline
\end{tabular}

" - sum of points according patient's diary; ${ }^{\nabla}-$ sum of points according CPI; $\Omega$ - sum of points according VPI;

In order to compare all $\mathrm{CP}$ clinical and additional research indicants between groups, a covariance dispersion analysis (ANCOVA) was used, filtering patient age, disease duration and average analysed indicant pretreatment value, with an aim to conclude, if the average indicant post-treatment value is affected by its belonging to research group in a statistically valid way. In order to define the difference between statistically valid groups, Post-hoc Sidac analysis was applied. The comparison of CP clinical and additional research indicators between groups is shown in Table 3.5. 


\subsection{CPI and VPI validity}

In statistical analysis CPI and VPI validity was defined. Both methods included construct and empirical validity analysis. In order to define CPI and VPI construct validity, Cronbach's Alpha test coefficient of internal consistency was applied. CPI Cronbach's Alpha coefficient reached 0.68. Further analysis showed that the exclusion of Question Nr. 3 (number of hospitalizations due to $\mathrm{CP}$ in anamnesis) from CPI seriously and positively impacts CPI Cronbach's Alpha coefficient, increasing it to 0.71. VPI Cronbach's Alpha coefficient reached 0.66. After exclusion of Question Nr. 2 (the echotexture of pancreas) the coefficient reached 0.70 .

CPI and VPI content empirical validity was defined ascertaining its correlation with quality of life indicators. Before treatment CPI of all patients substantially correlates with such EORTC QLQ-C30 indicators as general health and quality of life $(\mathrm{GH})(\mathrm{r}=-0.345 ; \mathrm{p}<0.05)$, functional scale point sum (FU) ( $\mathrm{r}=-0.467 ; \mathrm{p}<0.05)$, symptom scale point sum (SY) $(\mathrm{r}=0.532 ; \mathrm{p}<$ $0.001)$ and QLQ-PAN26 point sum $(r=0.595 ; \mathrm{p}<0.001)$.

CPI of all patients after treatment substantially correlates with such EORTC QLQ-C30 indicators as general health and quality of life $(\mathrm{GH})$ $(\mathrm{r}=-0.595 ; \mathrm{p}<0.001)$, functional scale point sum (SY) $(\mathrm{r}=-0.558 ; \mathrm{p}<0.001)$, symptom scale point sum (SY) $(r=0.566$; $\mathrm{p}<0.001)$ and QLQ-PAN26 point sum $(r=0.737 ; \mathrm{p}<0.001)$. CPI correlates with abdominal pain extent from patient's diary data after treatment $(\mathrm{r}=0.403 ; \mathrm{p}<0.001)$. VPI shows a considerable correlation with EORTC QLQ-C30 indicators - general health and quality of life $(\mathrm{GH})(\mathrm{r}=-0.362 ; \mathrm{p}<0.05)$ and symptom scale point sum (SY) after treatment $(\mathrm{r}=0.309 ; \mathrm{p}<0.05)$.

While analysing the results of research, CPI prognostic value was defined using linear regression analysis - it is possible to forecast CPI decrease after treatment depending on initial disease severity according to CPI and 
therapy applied. A linear regression model equation was obtained (for explanations see Tab. 3.6.):

$\mathrm{CPID}=-5.78+2.90 \times \mathrm{TG}+3.05 \times \mathrm{CPIG}$

where:

CPID - CPI decrease after treatment (\%);

TG - therapy group;

CPIG - CPI group.

Table 3.6.

\section{Predicted P, PP and PNP therapy effectiveness, using CPI}

\begin{tabular}{|c|c|c|c|}
\hline Therapy group & Therapy & CPI group * & $\begin{array}{c}\text { Predicted therapy } \\
\text { effectiveness }^{\Psi}\end{array}$ \\
\hline 1. & P & 1 & 0.62 \\
\hline 1. & P & 2 & 3.22 \\
\hline 1. & P & 3 & 6.27 \\
\hline 2. & PP & 1 & 3.07 \\
\hline 2. & PP & 2 & 6.12 \\
\hline 2. & PP & 3 & 9.17 \\
\hline 3. & PNP & 1 & 6.42 \\
\hline 3. & PNP & 2 & 9.02 \\
\hline 3. & PNP & 3 & 12.07 \\
\hline
\end{tabular}

* - CPI: group 1-6-8 points; group 2-9-15 points; group 3-16-25 points;

$\psi$ - predicted CPI decrease after one month long therapy

The applied medical therapy was not effective for six CP patients whose VPI before treatment reached 15 points and more, because after therapy course strong abdominal pain persisted. The further watch of those patients showed indications for surgical intervention of CP. Four patients had undergone surgical procedures, while two other patients refused for various reasons. For those patients who had surgical treatment, QoL function scale point sum increased and $\mathrm{CP}$ symptom scale point sum decreased two months after surgical intervention. The patients who refused from surgical intervention did not have any changes of QoL indicators after two months. The usefulness 
of VPI for the choice of surgical intervention in case of CP was assessed. On the 22nd of February, 2012, VPI was registered as the patent of the Republic of Latvia - "The surgical intervention indications assessment method in the case of chronic pancreatitis" (LV 14515 B) [38].

\subsection{Quality of life}

The $\mathrm{P}$ group statistically improved following EORTC QLQ-C30 indicators: the degree of symptoms decreased (SY) $(p=0.017)$; nausea and vomiting decreased $(\mathrm{NV})(\mathrm{p}=0.046)$; general health increased $(\mathrm{GH})$ $(\mathrm{p}=0.033)$; and following QLQ-PAN26 indicators: bowel disorders decreased $(\mathrm{AB})(\mathrm{p}=0.016)$ and muscle weakness decreased $(\mathrm{MW})(\mathrm{p}=0.039)$.

The PP group statistically improved following EORTC QLQ-C30 indicators: the total sum of points from the functional scales increased (FU) $(\mathrm{p}<0.001)$; the symptom degree decreased (SY) $(\mathrm{p}<0.001)$, physical functioning increased $(\mathrm{PF})(\mathrm{p}<0.001)$, as well as role functioning (RF) $(\mathrm{p}<0.001)$, emotional functioning $(\mathrm{EF})(\mathrm{p}=0.013)$, cognitive functioning $(\mathrm{CF})$ $(\mathrm{p}=0.002)$, social functioning $(\mathrm{SF})(\mathrm{p}<0.001)$, appetite (AP) $(\mathrm{p}<0.001)$, and general health $(\mathrm{GH})(\mathrm{p}<0.001)$; fatigue decreased (FA) $(\mathrm{p}<0.001)$, as well as nausea/vomiting (NV) $(\mathrm{p}<0.001)$, abdominal pain (PA) $(\mathrm{p}<0.001)$, and sleep disorders $(\mathrm{SL})(\mathrm{p}=0.001)$.

The PNP group statically improved all EORTC QLQ-C30 indicators $(p<0.05)$. The main quality of life indicants comparison between study groups is presented in table 3.7 . 
Table 3.7.

Quality of life changes significance level comparison between study groups

\begin{tabular}{|l|c|c|c|c|}
\hline \multirow{2}{*}{ Indicant } & \multicolumn{2}{|c|}{$\begin{array}{c}\text { Difference between indicant average } \\
\text { before and after treatment }\end{array}$} & $\begin{array}{c}\text { Three groups } \\
\text { comparison } \\
\text { (ANCOVA test, } \\
\text { p value) }\end{array}$ \\
\cline { 2 - 5 } & group P & group PP & group PNP & $<0.001$ \\
\hline $\mathrm{GH}^{\psi}$ & $-15.89 *$ & $-29.62 * *$ & $-38.87 * *$ & $<0.001$ \\
\hline $\mathrm{FU}^{\psi}$ & -54.77 & $-73.50 * *$ & $-158.17 * *$ & 0.001 \\
\hline SY & $146.11 *$ & $151.75^{\psi} * *$ & $270.22 * *$ & 0.003 \\
\hline PAN26 sum of points & 167.58 & $242.75^{*}$ & $449.69 * *$ & \\
\hline
\end{tabular}

$*-\mathrm{p}<0.05 ; * *-\mathrm{p}<0.001 ;{ }^{*}-$ the scale of EORTC QLQ-C30

In order to define the most effective option among used medications, as part of the given research about CP combined and medical therapy, 53 clinical, additional research and quality of life indicators were defined for 106 patients before and after one month long treatment course.

Summarizing the results of the research, it must be said that the patients who belonged to pancreatin monotherapy group experienced a statistically valid improvement of 14 indicators - abdominal pain decreased in opinion of both patient and doctor, appetite improved, WBC, alpha amylase, ALAT, ASAT and blood urea levels reduced, as well as EORTC QLQ-C30 showed the reduction of nausea and vomiting, bowel movement disorder, muscle weakness reduction and improvement $\mathrm{CP}$ symptom severity in general (EORTC QLQ-C30 and QLQ-PAN26).

In pancreatin and PPI combined therapy groups patients experienced a statistically valid improvement of 38 indicators - abdominal pain decreased in opinion of both patient and doctor, bowel movement disorder (according to patient's diary) and steatorrhea, ESR decreased, WBC, alpha amylase, ALAT, ASAT levels, glucose intolerance degree, total clinical pancreatic index - all decreased, as well as improvement of the following parameters was noticed: physical, role, emotional, cognitive, social and general functioning and global 
health indicators, muscle weakness, nausea and vomiting, pancreatic pains, sleep disorders, appetite loss, diarrhoea, CP symptoms in general and patient financial difficulties extent (EORTC QLQ-C30 and QLQ-PAN26).

In pancreatin, PPI and NSPL combined therapy group patients had improvement of 43 indicators - BMI increased, abdominal pain decreased in opinion of both patient and doctor, bowel movement disorder and appetite loss reduced (according to patient's diary), steatorrhea, ESR, WBC, alpha amylase levels, overall clinical and visual pancreatic index decreased. After treatment the patients of the given group experienced the following EORTC QLQ-C30 indicator improvements: physical, role, emotional, cognitive, social functioning, overall functioning and global health indexes; muscle weakness, nausea and vomiting, pancreatic pains, dyspnoea, sleep disorders, appetite loss, constipation, diarrhoea, general $\mathrm{CP}$ symptoms and patient financial difficulties extent reduced, as well as the following QLQ-PAN26 indicators decreased: pancreatic pain, bowel movement disorders, abdominal bloating, taste alterations, flatulence, body mass loss, muscle weakness, dry mouth, anxiety of health in the future and QLQ-PAN26 point sum, as well as digestive function, own body perception (body image aspects), sexual function and life planning capabilities improved.

After comparing the researched medical therapy impact on CP patient health indicators (ANCOVA), it was stated that pancreatin, PPI and NSPL combined therapy group after treatment, compared to pancreatin monotherapy and pancreatin and PPI combined therapy groups, showed the most apparent improvement of the following 13 indicators: BMI increased, abdominal pains (in view of patient and doctor) and bowel movement disorders (according to patient diary) reduced, clinical pancreatic index reduced and quality of life indicators improved - emotional and cognitive functioning, overall function and global health improved (EORTC QLQ-C30), as well as pancreatic pains, 
indigestion, anxiety of health in the future (QLQ-PAN26) and QLQ-PAN26 point sum.

The analysis of research results shows that no advantages of pancreatin monotherapy and pancreatin and PPI combined therapy were discovered in any analysed indicant, compared to pancreatin, PPI and NSPL combined therapy.

\subsection{Work approval}

The work was approved on 12th of June, 2013, during a meeting of Rīga Stradinš University Department of Internal Diseases, as well as presented on 14th of October, 2012, during XX European Gastroenterology Week (The Netherlands, Amsterdam), on 16th of October, 2013, during XXI European Gastroenterology Week (Germany, Berlin), on 12th of November, 2011, during V Latvian Gastroenterology congress with international participation (Rīga), on 20th of April, 2012, during International Conference in Pharmacology (Rīga), as well as during scientific conferences of RSU in 2010, 2011, 2012 and 2013. 


\section{DISCUSSION}

In treating chronic pancreatitis $(\mathrm{CP})$, at least two problems are relevant - lack of trusted methods of evaluation of clinical course severity and disease management efficacy and lack of widely accepted medical treatment protocol/approach. The aim of the study was to develop new assessment methods of chronic pancreatitis (CP) clinical course severity and pancreas structural change severity, and using pancreatin as monotherapy and as combination with proton pump inhibitor (PPI), as well as a combination of pancreatin, PPI and NSAID in patients with CP, to define the most effective medication treatment way among the ones applied. In given prospective randomized controlled study in order to appraise CP clinical course and pancreas structural change severity, some special methods were developed Clinical pancreatic index (CPI) and Visual pancreatic index (VPI). In the study CPI and VPI were used for CP patients in case of medical treatment. Patients with CP not needing surgical or endoscopic treatment were divided in three groups, depending on the type of therapy: 1) pancreatin monotherapy group, 2) pancreatin and PPI combined therapy group and 3) pancreatin, PPI and NSAID combined therapy group. Two study groups - pancreatin monotherapy group and pancreatin with PPI combined therapy group - were presented as the control group for pancreatin, PPI and NSAID combined therapy group. Efficacy of each medical treatment group was evaluated and compared by studying CP patient anamnesis, as well as evaluating degrees of abdominal pain, bowel movements disorder and appetite loss, laboratory tests and radiological investigations parameters.

Difficulties in CP diagnosis, thus in prediction of treatment and therapy outcome, may be explained through different morphologic types of the disease. In recent decades, it is thought to define two CP morphologic forms large and small duct CP. Although large and small duct CP may have similar 
symptoms, these morphological forms require a different approach in therapy [25]. Medical treatment is mostly effective in small duct CP. It is known that in case of small duct $\mathrm{CP}$, the effectiveness of pancreatic enzyme replacement therapy in managing abdominal pain is up to $70 \%$, but in case of large duct $\mathrm{CP}$ it is only $25 \%$. In author study it is noted that the major part of study patients (84.5\%) were presented with small duct $\mathrm{CP}$ and their ductus pancreaticus diameter was $3.5 \mathrm{~mm}$ and less.

Study patients sex ratio was $1.79(64.2 \%$ - males and $35.8 \%-$ females). Such male to female ratio is different from similar research sex ratio. Joergensen and colleague study revealed that the frequency ratio of men to women was 1,27 (56\% - men, 44\% - women) [39], but in Lévy study - 5.07 [2]. According to literature alcohol induced $\mathrm{CP}$ makes up even $50 \%-70 \%$ of the cases and is more frequent among male patients [40], whereas the female patients more commonly have an idiopathic CP [41]. In recent years there has been an observed decrease in the prevalence of alcohol induced chronic pancreatitis in developed countries. A study from Japan, published in 2000, showed that the prevalence of alcohol induced CP has decreased to $58.7 \%$ among male patients and to 55.5\% among female patients with CP [41], it corresponds to the data acquired by Joergensen study [39]. Alcohol etiology of the disease was found in 48 patients (55\%). In the author's study the alcohol etiology of the disease was found in $59.4 \%$ of patients $-70.6 \%$ of men and $39.5 \%$ women, suggesting that the prevalence of alcohol-induced chronic pancreatitis among Latvian men is still high.

One of CP etiologic factors is tobacco smoking. In the author's study $50.9 \%$ of the study patients were smokers $-67.7 \%$ of men and $18.4 \%$ women. Despite the tobacco control measures in Latvia, smoking prevalence is high [8]. The mean age of the study patients is 53.3 years, which corresponds to the literature data [2]. 
Abdominal pain is one of the hallmark symptoms of $\mathrm{CP}$, and in this study $97.2 \%$ patients noted its presence. There still is no opinion on the most accurate CP abdominal pain evaluation method [14]. In clinical study a visual analogue scale (VAS) is used frequently. One of the most precise methods of $\mathrm{CP}$ abdominal pain evaluation is an assessment of four signs - pain episode frequency, pain intensity (also using VAS), necessity of using NSAIDs and inability to work because of the CP. In author study, such method could not be used as group PNP patients were taking NSAID with analgesic effect and many patients were unemployed because of reasons other than $\mathrm{CP}$. To evaluate abdominal pain severity both the opinion of the patient and the judgment of the treating physician were taken into consideration. Patient's opinion was evaluated according to the patient diary data, by using both the modified VAS and several questions from the quality of life questionnaires (questions $\mathrm{Nr} .9$ and 19 of the EORTC QLQ-C30 and questions Nr. 33 and 34 of the QLQPAN26). The judgment of treating physician was used to fill the modified VAS independently of patient's opinion.

FE-1 detection with ELISA polyclonal antibody method was used to evaluate pancreatic exocrine function. This method allows to approve severe and moderate pancreatic exocrine insufficiency, but, due to a upper sensitivity threshold $-500 \mu \mathrm{g} / \mathrm{g}$, it did not allow to assess FE-1 dynamics under therapy in 7 patients with preserved pancreatic exocrine function with FE-1 levels higher than $500 \mu \mathrm{g} / \mathrm{g}$. Therefore, FE-1 assessment is an appropriate method in defining moderate to severe pancreatic exocrine insufficiency, but not suitable in case of mild exocrine insufficiency and in evaluation of medicinal therapy effects on pancreatic exocrine function in patients with preserved exocrine function.

The evaluation of the efficiency of medication therapy for patients with CP is complicated. There is no single and safe CP therapy efficiency criterion. It is necessary to consider many symptoms of the disease including subjective symptoms (abdominal pain, appetite loss), as well as indicators of 
many laboratory tests and other kinds of tests. Current classification of CP is not convenient and exact for evaluation of the clinical course of the disease in the clinical practice. In order to choose the right treatment for $\mathrm{CP}$, to evaluate the efficiency of it, and to predict the course of the disease, an instrument is necessary that would allow assessing the activity of the disease. This is not a new medical approach. For example, there are levels of severity for bronchial asthma, rheumatoid arthritis, an autoimmune orbitopathy, and many other diseases. In the case of $\mathrm{CP}$, a classification is necessary that would allow for a quantitative or semi-quantitative assessment of the most important symptoms of the disease.

In order to make a quantitative assessment of the severity of $\mathrm{CP}$, a Clinical pancreatic index (CPI) was developed for this study. This index helps to evaluate and sum up such indications of $\mathrm{CP}$ as the severity and number of surgical procedures due to $\mathrm{CP}$, the number of hospitalizations because of exacerbation/flare of $\mathrm{CP}$, the loss of weight, intensity of pain, degree of steatorrhoea, the number of daily defecations, other dyspeptic complaints, and impaired glucose tolerance. The results of the study show that CPI is valid instrument. The total CPI correlates with major QoL indicators, and CPI objectively reflects the severity of the clinical course of $\mathrm{CP}$. In this study analyzing the outcomes of various types of medical therapy, it was possible to determine the prognostic value of the CPI: it is possible to predict the improvement of the CP clinical course depend on the initial CPI and the type of treatment. Further research is needed in order to identify additional predictors of the drug therapy efficiency of chronic pancreatitis.

In comparing the dynamics of CPI among study groups, it was stated that the PNP treatment for patients with CP is more effective than pancreatin monotherapy or pancreatin and PPI combination. The author of the study believes that the CPI method can be useful in CP clinical research that aims not only to evaluate the efficiency of medical treatment but also that of endoscopic 
and surgical treatment. The use of CPI can provide a chance to develop common recommendations for the treatment of $\mathrm{CP}$, depending on severity of the course of the disease. In clinical practice the CPI can be a useful method for observing the course of $\mathrm{CP}$ - the use of CPI can help to confirm if treatment is inefficient as well as whether an alternate treatment should be considered.

In order to evaluate the severity of the structural change in the pancreas, a Visual pancreatic index (VPI) was developed. VPI quantitatively shows the pancreas' visual parameters that are characteristic to $\mathrm{CP}$ : pancreas head size, pancreas echotexture, calcinates, ductus pancreaticus diameter, pseudocysts, peripancreatic fibrosis, irregular pancreas contours, and concrements in the lumen of ductus pancreaticus. VPI does not depend on certain imaging technique of the pancreas - to calculate the VPI it is possible to use data from abdominal USG, EUS and CT. During the study, only in the PNP patient group, the VPI decreased with statistical significance that can be explained by the reduction of inflammation in the pancreatic parenchyma which was not observed in other groups. That means that the PNP treatment can also may improve the morphological features of CP. In this study a satisfactory correlation was observed between VPI and EORTC QLQ-C30 data - a negative correlation with general health after the treatment and a positive correlation with a degree of symptoms after the treatment. Study data indicates a weak and insignificant but still negative correlation between VPI and the intensity of abdominal pain before and after the treatment. It is not paradoxical as it is known that in the late stages of $\mathrm{CP}$, in the case of pancreatic burnout syndrome, abdominal pain can reduce even though pancreas morphological features are significant. This observation can indicate that VPI shows the stages of morphological changes in the pancreas rather but not the severity of the clinical course of the disease. It is known that in the late stages of CP the development of a segmental portal hypertension, duodenal and distal bile duct stenosis, exocrine and endocrine insufficiency become more relevant. 
Considering this, VPI can be useful in assessing the indications of the surgical intervention in the case of CP. On February 22, 2012, the VPI was registered as the patent of the Republic of Latvia - "The surgical intervention indications assessment method in the case of chronic pancreatitis" (LV 14515 B) [38]. The VPI is an informative, safe, and easy to reproduce method that does not require invasive investigation and the hospitalization of a patient.

Treatment of $\mathrm{CP}$ is a hard task. A characteristic of this disease is abdominal pain that is resistent to therapy, irreversible loss of pancreatic parenchyma due to fibrosis and development of severe chronic complications. Several CP abdominal pain mechanisms are known - increase in pressure in pancreatic ducts and parenchyma, inflammation, pancreatic tissue autolysis, pancreatic nerve fiber infiltration with immune cells as well as compression of pancreatic parenchyma, nerve, ganglia and duodenum, pancreatic tissue ischemia, meteorism etc. Without pain, CP patient wellness and working capacity is influenced by fatigue, weight loss, abnormal bowel movements, loss of appetite and other digestive disorders. Pacients with $\mathrm{CP}$ usually have lowered QoL with reduced physical, role, emotional and social performance. A medical treatment remains the main $\mathrm{CP}$ curing way. The main $\mathrm{CP}$ medical treatment method is the use of pancreatins.

Pancreatic exocrine secretion is regulated by means of feedback [ 26 , 42]. Pancreatic proteases, reaching the duodenum, cleave CCK-releasing peptide and thus lower serum CCK and pancreatic enzyme secretion stimulation. This means that $\mathrm{CP}$ patients undergoing pancreatin treatment may hope for reduction of pancreatic duct and parenchyma pressure and tissue autolysis, which, in turn, reduces abdominal pain.

There are several studies about the analgesic effect of the pancreatins which yielded conflicting results, but meta-analysis concluded that they are not effective for relieving abdominal pain in case of CP [17]. The conclusion could be explained by several factors. 
First, the patient populations were very heterogeneous in terms of both the etiology and the severity of the disease as well as the duration of the treatment.

Second, different pancreatins were used in these studies - enteric coated and non-enteric coated. In studies that used enteric coated pancreatins, analgetic effect of pancreatins was not observed [21, 22, 23, 24], but in studies that used non-enteric coated pancreatins the effect was observed $[18,19]$. It is known that pancreatic juice proteases have an inhibition effect on pancreatic ferment production only from within the proximal small intestine lumen. The main condition for enzymes to be released from enteric microcapsules is duodenal $\mathrm{pH}$ - enzymes are freed if $\mathrm{pH}$ is around 5.5-6.0. It is also known that patients with CP may have decreased bicarbonate production and impaired gastrointestinal tract motor activity. This may probably explain why pancreatins from enteric microcapsules could have been freed outside of the duodenum - in jejunum or ileum, and pancreatic exocrine secretion inhibition and pain relief was not possible. Combining pancreatin with PPI, we may hope that duodenal $\mathrm{pH}$, reaching sufficient levels, allows for pancreatin release from microcapsules inside duodenal lumen. This possibly provides an opportunity for a more efficient pancreatic exocrine function inhibition and significant reduction of abdominal pain. Author study gave an opportunity to compare pancreatin monotherapy and pancreatin in combination with PPI effect on abdominal pain in CP patients. Study results show that patients that were given pancreatin with PPI combined therapy noted more pronounced pain reduction than patients receiving pancreatin monotherapy (QLQ-PAN26 pancreatic pain indicant).

Third, three out of four studies that showed a lack of effectiveness of the pancreatin for the treatment of abdominal pain in $\mathrm{CP}$ had short treatment duration - from one to two weeks, what can be insufficient to achieve the desired effect. Generally, in order to judge the effect of pancreatin on 
abdominal pain caused by $\mathrm{CP}$, a month-long course of treatment is recommended.

Fourth, the above cited studies used high doses of pancreatins, which exceeded the recommended dosage for the treatment of steatorrhea. In Mössner study patients received 10000 IU (European Pharmacopoeia units) of proteases/day [24], whereas the patients in this study just $3750 \mathrm{IU} /$ day (European Pharmacopoeia units). And it has shown that high doses of proteases instead of decreasing the exocrine secretion of pancreas, rather stimulate it, thereby provoking secretion of CCK and in such a way increasing pain [43].

Fifth, interesting enough that in all studies which showed a lack of effectiveness of pancreatin for the treatment of abdominal pain in $\mathrm{CP}$ there was a paradoxically high placebo effect - up to $40 \%[21,22,23,24]$, despite the fact that frequently such pain is treatment resistant. Several causes are proposed for the exceptionally high placebo effect. Most of the studies have a crossover design, which lacked a wash-out period, thereby artificially increasing the placebo effect $[21,23,24]$. An exceedingly high placebo effect can be observed when the subjective parameters like pain and depression are evaluated, whereas in cases of bacterial infection, infertility and hyperglycemia the placebo effect is much milder [44]. But a more recent study of abdominal pain due to $\mathrm{CP}$ concluded that the placebo effect on abdominal pain is lower - up to $20 \%$ [45]. This fact possibly didn't allow to prove the effect of pancreatin in decreasing the abdominal pain in previous studies, which covered enteric coated pancreatin effect on CP abdominal pain. In this study analgesic effect of pancreatin $37 \%$ $(\mathrm{p}=0.008)$ was observed.

The existing guidelines for treatment of $\mathrm{CP}$ recommend the use of PPIs only in cases of steatorrhea to increase the effect of pancreatin. But when pathogenesis of $\mathrm{CP}$ is taken into consideration, PPIs could be useful not only to decrease the secretion of gastric acid but also to decrease the exocrine secretion of pancreas, as PPIs can block the $\mathrm{H}^{+} / \mathrm{K}^{+}$-ATP-ases of pancreas duct cells [27]. 
As a result, a decline of bicarbonate and water production in pancreas ducts can be expected with subsequent reduction of abdominal pain. In this study the combination of PPI and pancreatin was more effective for decreasing the abdominal pain, abnormal bowel movements and for improving the quality of life, when compared to pancreatin therapy alone. Thereby this study shows that, in addition to previously published guidelines, the PPIs can have a wider application for treatment of CP. Author chose omeprazole, because this is the most often used medication, and it is cheaper and more available compared to other PPIs. According to the recommendations of PPI usage, the cheapest PPI should be used [34]. Concerning the dosage, it must be noted that in the case of $\mathrm{CP}$ the dose of omeprazole is not defined. The most often used dose is $20 \mathrm{mg}$ once a day. It is used in the cases of gastric and duodenal ulcers (including NSAID-induced) but in cases when more distinct $\mathrm{HCl}$ production suppression is needed, the dose of omeprazole is bigger. For example, in the case of erosive esophagitis or $\mathrm{H}$. pylori eradication therapy, the dose of omeprazole is $20 \mathrm{mg}$ twice a day. Although it is known that the $20 \mathrm{mg}$ dose of omeprazole once a day ensures the suppression of $\mathrm{HCl}$ secretion up to $97 \%$, there is also data that the $20 \mathrm{mg}$ dose of omeprazole twice a day ensures better gastric $\mathrm{pH}$ profile during the night. If omeprazole is used at $20 \mathrm{mg}$ twice a day, the periods with gastric $\mathrm{pH}>3$ and $>4$ are significantly longer compared to usage of the $20 \mathrm{mg}$ dose of omeprazole once a day [46].

PPIs have many tasks in CP treatment. In this study the aim of using PPI was to suppress the production of gastric $\mathrm{HCl}$, to inhibit the production of bicarbonate and water in the ducts' cells, protect the pancreatin from inactivation due to $\mathrm{HCl}$ effects and to protect gastrointestinal mucosa from possible NSAID-induced damage. It may be possible that all these aims can be achieved with a lesser dose of omeprazole. Further studies are necessary to find the optimal dose of PPI for CP patients. 
Most of the national guidelines include the NSAIDs as a treatment for $\mathrm{CP}$ abdominal pain $[1,14,15,16]$. But a long term usage of NSAIDs is not recommended in cases of chronic pain due to possible side effects, although the frequency of such side effects in cases of CP has not been studied. One study showed that sulindac decreased both the fibrosis of pancreas and the parenchymal infiltration with immune cells in a mouse model CP [30]. NSAIDs can decrease the risk of both pancreas and gastrointestinal cancer. The data from literature indicates that NSAID may have serious side effects but they differ significantly among different preparations [47] and patients from different age groups; the side effects are higher for patients with a prior history of gastrointestinal events and higher if corticosteroid has been used. The risks also depend on the dosage of NSAID, the primary disease, and duration of treatment. The common concerns about conventional NSAIDs side effects on gastrointestinal tract partially stem from the frequent use of these medications without any kind of gastric protection, and from the fact that the prescription guidelines are frequently ignored. Studies have shown that the selective COX-2 inhibitors are much less toxic for gastrointestinal tract. However, selective COX-2 inhibitors have a higher risk of cardiovascular events compared to nonselective COX inhibitors.

In this study the Aceclofenac was used as a NSAID, although aceclofenac is not really a selective COX-2 inhibitor, since it mainly inhibits COX-2. Aceclofenac is one of the safest NSAIDs where gastrointestinal side effects are concerned [48]. In order to reduce the possible side effects of aceclofenac, the following exclusion criteria were used in this study: gastric and duodenal ulcer, severe liver and kidney disease, acute cerebral and myocardial infarction and severe cardiac failure. All patients went through fibrogastroscopy before the therapy. In this study the manufacturer's recommended dose of aceclofenac was used for 30 days, which is considered short term therapy. To see how safe it would be to use the aceclofenac for each 
of the patients, several tests were made before and after PNP treatment to assess the level of hemoglobin, RBC, ALAT, ASAT and blood urea level as well as occult blood in feces. Before the treatment mild anemia was observed in six out of 20 patients in the $\mathrm{P}$ group, three out of 48 in the PP group, and three out of 38 in the PNP group. In all groups the level of hemoglobin was not changed after the treatment, and it also did not differ after the treatment when comparing all three groups. A new case of anemia after the treatment was detected only in one case, only in the $\mathrm{P}$ group. Before the treatment occult blood in feces were detected in 3 patients in the $\mathrm{P}$ group, and 2 patients in the PNP group, but after the treatment occult blood in feces were detected only in one patient in both the $\mathrm{P}$ and PNP groups. Both of these patients were diagnosed hemorrhoids later on. After the treatment, the level of ALAT and ASAT decreased in groups $\mathrm{P}$ and PP $(\mathrm{p}<0.05)$ but it remained unchanged in the PNP group. After the treatment, the blood urea level in the pancreatin monotherapy group decreased, but in the other groups - it did not change. The cardiovascular events were not observed during the research. A one month long course of PNP therapy in patients with CP was safe and didn't cause any significant side effects. In order to diminish the possible side effects of aceclofenac, further studies would be necessary. They would allow researchers to clarify whether a lower dose of aceclofenac in the PNP therapy has the same efficiency also in the patients with CP.

After the therapy, BMI was statistically significantly higher only in PNP group. Decrease in BMI in patients with $\mathrm{CP}$ could be connected to loss of appetite and nutrient malabsorption. Unfortunately, study groups didn't differ in loss of appetite after the therapy. During the research, significant improvement in appetite was noted in P and PNP groups. Appetite may be a part of hunger. It is well known that CP patients retain appetite, though patients may suffer from postprandial pain. In order to avoid such pain, patients limit their food intake and frequency, which leads to reduction in body mass. 
Although P and PP therapy intended improvement in nutrient absorption in CP patients, PNP therapy more efficiently helped with abdominal pain, thus the increase in patient BMI. Patients in PNP group had better emotional performance after treatment comparing to $\mathrm{P}$ and $\mathrm{PP}$ groups, which may have significantly improved appetite and BMI in these patients. In the disease exacerbation periods patients may have nausea, vomiting and intoxication, which may be a cause of appetite loss. Unfortunately, study groups were not different in nausea and vomiting degrees (using EORTC QLQ-C30 NV) after therapy.

The results of this study show that for reduction of abdominal pain and abnormal bowel movements in patients with CP PNP therapy is more effective than treatment with pancreatin alone or than treatment with a combination of pancreatin and a PPI. In addition, such treatment also increases the BMI and improves the clinical course of the disease and the quality of life. PNP therapy of CP is rational, as it's based on pathogenesis of the disease, effective, safe and economically advantageous. 


\section{CONCLUSIONS}

1. The Clinical pancreatic index as an integral objective indicator is valid, and reflects the severity of the chronic pancreatitis course, and the efficiency of medical treatment for patients with chronic pancreatitis.

2. The Clinical pancreatic index has prognostic significance in choosing medical treatment with pancreatin alone, a combination of pancreatin and proton pump inhibition or a combination of pancreatin, proton pump inhibition, and a non-steroidal anti-inflammatory drug.

3. The Visual pancreatic index shows the severity of the pancreas' structural changes, and predicts the necessity for surgical treatment in the case of chronic pancreatitis.

4. The combination of pancreatin, a proton pump inhibitor and a nonsteroidal anti-inflammatory drug is the most effective medical treatment for patients with chronic pancreatitis, regardless of the severity of the course of the disease, when compared to the pancreatin monotherapy and pancreatin in combination with a proton pump inhibitor.

5. The combination of pancreatin, a proton pump inhibitor and a nonsteroidal anti-inflammatory drug is the most effective medical treatment for patients with chronic pancreatitis, in reducing abdominal pain, bowel movements, and steatorrhoea, as well as for increasing the body mass index and improving the clinical course of the disease, compared to the pancreatin monotherapy and pancreatin in combination with a proton pump inhibitor.

6. The combination of pancreatin, a proton pump inhibitor and a nonsteroidal anti-inflammatory drug is most effective in increasing the quality of life for patients with chronic pancreatitis, compared to the 
pancreatin monotherapy and pancreatin in combination with a proton pump inhibitor.

7. A month long treatment with pancreatin, a proton pump inhibitor and a non-steroidal anti-inflammatory drug for patients with chronic pancreatitis is safe and does not cause a serious adverse reaction. 


\section{PRACTICAL RECOMMENDATIONS}

1. The Clinical pancreatic index shows the severity of the clinical course of chronic pancreatitis objectively, and predicts the efficiency of pancreatin, a proton pump inhibitor and a non-steroidal antiinflammatory drug, therefore the usage of this index is advised for this purpose.

2. The Visual pancreatic index shows the severity of the pancreatic parenchyma structural changes objectively, therefore the usage of this index is advised in evaluating the necessity of surgical treatment.

3. The combination of pancreatin, a proton pump inhibitor and a nonsteroidal anti-inflammatory drug is effective and advisable to patients with chronic pancreatitis. 


\section{ACKNOWLEDGEMENTS}

Cordial gratitude and hearty appreciation to my husband Aleksandrs Umnovs for care, understanding, support and constructive advices. A heartfelt thank you to my children Jelizaveta un Sergejs for support, patience and understanding.

I express my gratitude for supervising this study to Professor $D r$. habil. med. Grigorijs Orlikovs for encouragement, support and valuable advice.

Cordial gratitude to Professor Dr. med. Aivars Lejnieks, Head of RSU Department of Internal Diseases for support provided in the elaboration of this work.

I thank Dr. phys.docent Renārs Erts, Head of RSU Physics department for consultations during statistical data analysis.

A warm thank you goes to RSU Department of Internal Diseases Associated professor Dr. med. Julija Voicehovska and RSU Department of Internal Diseases Docent Dr. med. Vladimirs Voicehovskis for constructive advice and given support.

Many thanks to Pauls Stradins Clinical University Hospital Center of Gastroenterology doctors Vija Voltnere, Ilze Pḷavina and Ēvalds Ostrovskis for co-operation, worthy pieces of advice and discussions. I thank Head of Pauls Stradins Clinical University Hospital Center of Immunology Inta Jaunalksne for co-operation and support. I thank Viktorija Priedīte, Head of Immunology laboratory BIOCON for co-operation and constructive advice.

I thank Rīga Stradiņš University and European Social Fund for financial support in this study. I thank companies BERLIN-CHEMIE, AS OlainFarm and Gedeon Richter for provision of Pangrol 25 000, Omeprazol and Airtal in the form of humanitarian aid.

I express my gratitude to the editor of the RSU Publishing Division Aija Lapsa, for advice and assistance during the work. 


\section{CONFLICT OF INTEREST}

Promotional work was done with financial support of European Social Fund "Support for doctorates in acquiring study programme and obtaining scientific degree in Riga Stradins university" (Project agreement $\mathrm{Nr}$. 2009/0147/ 1DP/1.1.2.1.2/09/IPIA/VIAA/009) and support of BERLINCHEMIE, OlainFarm and Gedeon Richter - Pangrol 25 000, Omeprazol and Airtal (aceclofenac) were received in the form of humanitarian aid.

The protocol of study, the protocol of agreement, and the protocol of participation corresponded to the Helsinki declaration on principles of humanity in medicine and approved by Ethics committee of Riga Stradins university (Riga, Latvia) - E-9 (2), 11.06.2009.

The study respects the Law on personal data protection rules. Personal information obtained in the course of the research is strictly secured to avoid external leaks. Confidence is assured in the medicine ethics and scientific rigor. Informative sheets were elaborated to inform the study patients about the nature of the study. The protocol of agreement was signed by all study patients. 


\section{REFERENCES}

1. Danilāns A., Gardovskis J., Pokrotnieks J., et al. (2002) Hronisks pankreatîts. Diagnozes un ārstēšanas standarts. - Rīga: Gastroenterologijas Atbalsta fonds.

2. Levy P., Barthet M., Mollard B., et al. (2006) Estimation of the prevalence and incidence of chronic pancreatitis and its complications // Gastroenterol Clin Biol, 30:838-844.

3. Levy P., Milan C., Pignon J., et al. (1989) Mortality factors associated with chronic pancreatitis. Unidimensional and multidimensional analysis of a medical-surgical series of 240 patients // Gastroenterology, 96:1165-1172.

4. Gardner T., Kennedy A., Gelrud A., et al. (2010) Chronic pancreatitis and its effect on employment and health care experience: results of a prospective American multicenter study // Pancreas, 39(4):498-501.

5. Latvijas veselības aprūpes statistikas gadagrāmata 2011. gads, 13. izdevums. Slimību profilakses un kontroles centrs, 2012 // http://www. spkc.gov.lv/veselibas-aprupes-statistika/ (skatīts 16.09.2013.).

6. Stacionārā palīdzība. Statistikas dati par stacionāro gultu fondu un tā izmatošanas rādītājiem, ķirurğiskā darba rādītājiem Latvijas stacionāros un stacionāros ārstētajiem pacientiem 2010. gadā. Veselības ekonomikas centrs, 2010 // http:// vec.gov.lv/uploads/ files/4ecfde217b8b5.pdf (skatîts 16.09.2013.).

7. Alkohola patēriņš Latvijā. Pasaules Veselības Organizācija, 2013 // http://apps.who.int/gho/data/node.main.A1032?lang=en

(skatīts 16.09.2013.)

8. Tabakas smēķēšanas prevalence Latvijā. Pasaules Veselības Organizācija, 2000 // http://www.who.int/tobacco/media/en/Latvia. pdf (skatīts 16.09.2013.). 
9. Schneider A., Löhr J., Singer M. (2007) The M-ANNHEIM classification of chronic pancreatitis: introduction of a unifying classification system based on a review of previous classifications of the disease // J Gastroenterol, 42:101-119.

10. Uomo G. (2002) How far are we from the most accurate classification system for chronic pancreatitis? // J Pancreas (Online), 3(3):62-65.

11. Sarner M., Cotton P. (1984) Classification of pancreatitis // Gut, 25:756-759

12. Bloechle C., Izbicki J., Knoefel W., et al. (1995) Quality of life in chronic pancreatitis - results after duodenum-preserving resection of the head of the pancreas // Pancreas, 11:77-85.

13. Oza M., Kahaleh M. (2013) Endoscopic management of chronic pancreatitis // World J Gastrointest Endosc, 16;5(1):19-28.

14. Frulloni L., Falconi M., Gabbrielli A., et al. (2010) Italian consensus guidelines for chronic pancreatitis // Dig Liver Dis, 42(6):S381-406.

15. de-Madaria E., Abad-González A., Aparicio J., et al. (2013) The Spanish Pancreatic Club's recommendations for the diagnosis and treatment of chronic pancreatitis: part 2 (treatment) // Pancreatology, 13(1):18-28.

16. Warshaw A., Banks P., Fernández-Del Castillo C. (1998) AGA technical review: treatment of pain in chronic pancreatitis // Gastroenterology, 115(3):765-776.

17. Brown A., Hughes M., Tenner S., et al. (1997) Does pancreatic enzyme supplementation reduce pain in patients with chronic pancreatitis: a meta-analysis // Am J Gastroenterol, 92(11):20322035.

18. Isaksson G., Ihse I. (1983) Pain reduction by an oral pancreatic enzyme preparation in chronic pancreatitis // Dig Dis Sci, 28(2):97102. 
19. Slaff, J., Jacobson D., Tillman C., et al. (1984) Protease-specific suppression of pancreatic exocrine secretion // Gastroenterology, $87: 44-52$.

20. Domínguez-Muñoz J., Iglesias-García J., Iglesias-Rey M., et al. (2006) Optimising the therapy of exocrine pancreatic insufficiency by the association of a proton pump inhibitor to enteric coated pancreatic extracts // Gut, 55(7): 1056-1057.

21. Halgreen H., Pedersen N., Worning H. (1986) Symptomatic effect of pancreatic enzyme therapy in patients with chronic pancreatitis // Scand J Gastroenterol, 21(1):104-108.

22. Larvin M., McMahon M., Thomas W., et al. (1991) Creon (enteric coated pancreatin microspheres) for the treatment of pain in chronic pancreatitis: a double-blind randomized placebo-controlled crossover study // Gastroenterology, 100:A283.

23. Malesci A., Gaia E., Fioretta A., et al. (1995) No effect of long-term treatment with pancreatic extract on recurrent abdominal pain in patients with chronic pancreatitis // Scand J Gastroenterol, 30(4):392398.

24. Mössner J., Secknus R., Meyer J., et al. (1992) Treatment of pain with pancreatic extracts in chronic pancreatitis: results of a prospective placebo-controlled multicenter trial // Digestion, 53(1-2):54-66.

25. Yekebas E., Bogoevski D., Honarpisheh H., et al. (2006) Long-term follow-up in small duct chronic pancreatitis: A plea for extended drainage by "V-shaped excision" of the anterior aspect of the pancreas // Ann Surg, 244(6):940-946.

26. Owyang C., Louie D., Tatum D. (1983) Feedback regulation of pancreatic enzyme secretion suppression of cholecystokinin release by trypsin // Gastroenterology, 84:1268. 
27. Novak I., Wang J., Henriksen K., et al. (2011) Pancreatic bicarbonate secretion involves two proton pumps // J Biol Chem, 286(1):280-289.

28. Olsen T. (1978) The incidence and clinical relevance of chronic inflammation in the pancreas in autopsy material // Acta Pathol Microbiol Scand, 86A:361.

29. Whitcomb D., Barmada M. (2007) A systems biology approach to genetic studies of pancreatitis and other complex diseases // Cell Mol Life Sci, 64(14):1763-1777.

30. Bai H., Chen X., Zhang L., Dou X. (2012) The effect of sulindac, a non-steroidal anti-inflammatory drug, attenuates inflammation and fibrosis in a mouse model of chronic pancreatitis // $B M C$ Gastroenterol 24;12:115.

31. Hofbauer B., Saluja A., Lerch M., et al. (1998) Intra-acinar cell activation of trypsinogen during caerulein-induced pancreatitis in rats // Am J Physiol, 275:G352-362.

32. Graham D., Agrawal N., Campbell D., et al. (2002) Ulcer prevention in long term users of non-steroidal anti-inflammatory drugs: results of a doubleblind randomised, multicenter, active and placebo-controlled study of misoprostol versus lansoprazole // Arch Intern Med, 162:169175.

33. Hooper L., Brown T., Elliott R., et al. (2004) The effectiveness of five strategies for the prevention of gastrointestinal toxicity induced by nonsteroidal anti-inflammatory drugs: a systematic review // $B M J$, 329:948.

34. Guidance on the use of proton pump inhibitors in the treatment of dyspepsia. National Institute for Clinical Excellence, 2000 // http://www.nice.org.uk/guidance/ta7/resources/hta-report-on-protonpump-inhibitors-ppi-2 (skatīts 01.09.2014.) 
35. Fitzimmons D., Kahl S., Butturini G., et al. (2005) Symptoms and quality of life in chronic pancreatitis assessed by structured interview and the EORTC QLQ-C30 and QLQ-PAN26 // Am J Gastroenterol, 100: 918-926.

36. Shah N., Makin A., Sheen A., et al. (2010) Quality of life assessment in patients with chronic pancreatitis receiving antioxidant therapy // World J Gastroenterol, 16(32):4066-4071.

37. Witzigmann H., Max D., Uhlmann D., et al. (2002) Quality of life in chronic pancreatitis: a prospective trial comparing classical Whipple procedure and duodenum-preserving pancreatic head resection // $J$ Gastrointest Surg, 6(2):173-180.

38. Umnova L., Orļikovs G., Voicehovska J., Ivanova L. (2012) Ķirurg̣iskas iejaukšanās indikāciju noteikšanas paṇēmiens hroniska pankreatîta gadījumā // Latvijas Republikas patentu valdes oficiālais vēstnesis, Patenti un preču zīmes, 8:1130.

39. Joergensen M., Brusgaard K., Crüger D., et al. (2010) Incidence, prevalence, etiology, and prognosis of first-time chronic pancreatitis in young patients: a nationwide cohort study // Dig Dis Sci, 55(10):29882998.

40. Smith D., Burvill P. (1990) Relationship between male pancreatitis morbidity and alcohol consumption in Western Australia, 1971-84 // Br J Addict, 85(5):655-658.

41. Lin Y., Tamakoshi A., Matsuno S., et al. (2000) Nationwide epidemiological survey of chronic pancreatitis in Japan // $J$ Gastroenterol, 35(2):136-141.

42. Garces M., Gomez-Cerezo J., Codoceo R., et al. (1998) Postprandial cholecystokinin response in patients with chronic pancreatitis in treatment with oral substitutive pancreatic enzymes // Dig Dis Sci, 43(3):562-566. 
43. Mössner J., Wresky H., Kestel W., et al. (1989) Influence of treatment with pancreatic extracts on pancreatic enzyme secretion // Gut, 30(8):1143-1149.

44. Kirsch I. (2013) The placebo effect revisited: lessons learned to date // Complement Ther Med, 21(2):102-104.

45. Capurso G., Cocomello L., Benedetto U., et al. (2012) Meta-analysis: the placebo rate of abdominal pain remission in clinical trials of chronic pancreatitis // Pancreas, 41(7):1125-1131.

46. Geus W., Mulder P., Nicolai J., et al. (1998) Acid-inhibitory effects of omeprazole and lansoprazole in Helicobacter pylori-negative healthy subjects // Aliment Pharmacol Ther, 12(4):329-335.

47. Bombardier C., Laine L., Reicin A., et al. (2000) Comparison of upper gastrointestinal toxicity of rofecoxib and naproxen in patients with rheumatoid arthritis // N Engl J Med,343:1520-1528.

48. Llorente M., Tenías J., Zaragoza A. (2002) Comparative incidence of upper gastrointestinal bleeding associated with individual nonsteroidal anti-inflammatory drugs // Rev Esp Enferm Dig, 94(1):7-18. 


\section{LIST OF AUTHOR'S PUBLICATIONS}

1. Patents:

1.1. Umnova L., Orļikovs G., Voicehovska J., Ivanova L. (2012) Ķirurǵiskas iejaukšanās indikāciju noteikšanas paṇēmiens hroniska pankreatīta gadījumā // Latvijas Republikas patentu valdes oficiālais vēstnesis, Patenti un preču zīmes, 8:1130.

2. Cited publications:

2.1. Umnova L., Orļikovs G., Voicehovska J. (2011) Chronic pancreatitis: problems of classification // Acta Chirurgica Latviensis, 11/2:126129.

2.2. Umnova L., Orlikov G., Voitsekhovskaja Y., Voltnere V., Ostrovskis E., Voitsekhovskis V. (2011) Comparative investigation of quality of life and Clinical pancreatic index in patients with chronic pancreatitis // Therapeutic archives, (2):61-63.

2.3. Umnova L., Orḷikovs G., Voicehovska J., Voicehovskis V., Krustiņš E. (2014) Combined medical treatment of chronic pancreatitis // Proceedings of the Latvian Academy of Sciences, Section B, $68 ; 3(690): 20-30$.

3. Educational materials:

3.1. Umnova L., Orl̦ikovs G., Voicehovska J., Pl̦aviņa I. PNP therapy pathogenetic treatment of chronic pancreatitis // http://www.elearning.ueg.eu/documents-view.html?eprs\%5Br\%5D=18245

3.2. Umnova L., Orļikovs G., Voicehovska J., Pḷaviņa I. The therapeutic effect of PNP therapy in chronic pancreatitis clinical course improvement. Clinical pancreatic index // http://www.elearning.ueg.eu/documents-view.html?no_cache=1\&eprs\%5Br\%5D= 19116 
4. Articles:

4.1. Umnova L., Orḷikovs G., Voicehovska J., Voltnere V. (2010) Klīniskais pankreātiskais indekss un EORTC QLQ-C30 pacientiem ar hronisku pankreatītu // RSU Zinātniskie raksti, 1:2010: 64-69.

4.2. Umnova L., Orl̦ikovs G., Voicehovska J., Voltnere V., Pḷaviņa I. (2011) Fēču pankreātiskās elastāzes-1 līmen̦a dinamika hroniska pankreatīta kombinētās terapijas ietekmē // RSU Zinātniskie raksti, $1: 111-117$.

5. International conferences, congresses, workshops, meetings proceedings, books of abstracts:

5.1. Umnova L., Orl̦ikovs G., Voicehovska J., Voltnere V., Pḷaviņa I., Jaunalksne I. (2011) Fecal pancreatic elastase-1 dynamics in patients with chronic pancreatitis beyond combined treatment // V Latvian Gastroenterology congress with International Participation, Riga Stradinš University 10 th Scientific Conference, Abstract Book Supplement I: 12 .

5.2. Umnova L., Orḷikovs G., Voicehovska J., Pḷaviņa I. (2012) Pathogenetic basis of chronic pancreatitis PNP therapy // International Conference in Pharmacology, Rīga, Abstract Book: P28.

5.3. Umnova L., Orḷikovs G., Voicehovska J., Pḷaviņa I. (2012) PNP therapy - pathogenetic treatment of chronic pancreatitis // 20th United European Gastroenterology Week, Amsterdam, 2012, Gut, 61(3): A354.

5.4. Umnova L., Orļikovs G., Voicehovska J., Plaviņa I. (2013) The therapeutic effect of pnp therapy in chronic pancreatitis clinical course improvement. Clinical pancreatic index // 21st United European Gastroenterology Week, Berlin, 2013, United European Gastroenterology Journal, 1(1): A459. 
6. RSU conferences abstracts:

6.1. Umnova L., Orlikovs G., Voicehovska J., Voltnere V. (2010) Hroniska pankreatīta PNP terapijas patog்enētiskie aspekti // RSU ZK, Tēžu krājums, Rīga, II:104.

6.2. Umnova L., Orl̦ikovs G., Voicehovska J., Voltnere V., Pl̦aviņa I., Sardiko G. (2011) Dzīves kvalitātes novērtēšana pacientiem ar hronisku pankreatītu // RSU ZK, Tēžu krājums, Rīga, II:53.

6.3. Umnova L., Orļikovs G., Voicehovska J., Voltnere V., Pḷaviņa I. (2012) Pankreatīna un protona sūkṇa inhibitora kombinētās terapijas ietekme uz aikungga dziedzera eksokrīno sekrēciju pacientiem ar hronisku pankreatītu // RSU ZK, Tēžu krājums, Rīga, II:139.

6.4. Umnova L., Orḷikovs G., Voicehovska J., Pḷaviņa I. (2013) Hroniska pankreatīta patoǵenētiskā medikamentozā terapija // RSU ZK, Tēžu krājums, II:104. 